\title{
Pointwise Convergence of the Fractional Schrödinger Equation in $\mathbb{R}^{2}$
}

\author{
Chu-Hee Cho* and Hyerim Ko
}

\begin{abstract}
We investigate the pointwise convergence of the solution to the fractional Schrödinger equation in $\mathbb{R}^{2}$. By establishing $H^{s}\left(\mathbb{R}^{2}\right)-L^{3}\left(\mathbb{R}^{2}\right)$ estimates for the associated maximal operator provided that $s>1 / 3$, we improve the previous result obtained by Miao, Yang, and Zheng [19]. Our estimates extend the refined Strichartz estimates obtained by $\mathrm{Du}, \mathrm{Guth}$, and $\mathrm{Li}[10$ to a general class of elliptic functions.
\end{abstract}

\section{Introduction}

For $\alpha>1$, we consider the fractional Schrödinger equation

$$
i \partial_{t} u+(-\Delta)^{\alpha / 2}=0, \quad u(x, 0)=f(x)
$$

for $f \in H^{s}\left(\mathbb{R}^{2}\right)$. Here, $H^{s}$ is the $L^{2}$ Sobolev space of order $s$. Formally, the solution of (1.1) can be written as

$$
U_{\alpha} f(x, t)=(2 \pi)^{-2} \int_{\mathbb{R}^{2}} e^{i\left(x \cdot \xi+t|\xi|^{\alpha}\right)} \widehat{f}(\xi) d \xi .
$$

In this study, we investigate the order of $s$ for which

$$
\lim _{t \rightarrow 0} U_{\alpha} f(x, t)=f(x) \quad \text { a.e. } x
$$

holds whenever $f \in H^{s}\left(\mathbb{R}^{2}\right)$.

The problem of determining the optimal regularity $s$ for which 1.2 holds for the Schrödinger equation was initially studied by Carleson 6 . When $d=1$, he proved the convergence of 1.2 with $\alpha=2$ for $s \geq 1 / 4$, whereas it generally fails for $s<1 / 4$ in any dimension, as shown by Dahlberg and Kenig [9].

In higher dimensions, Sjölin 21] and Vega 24 independently showed that 1.2 with $\alpha=2$ holds for $s>1 / 2$. This result was improved to $s>1 / 2-1 /(4 d)$ by Lee 16 for $d=2$ and by Bourgain [2] for $d \geq 3$. Subsequently, Bourgain [3] showed that $s \geq d /(2 d+2)$ is

Received June 18, 2021; Accepted September 14, 2021.

Communicated by Sanghyuk Lee.

2020 Mathematics Subject Classification. 35Q41.

Key words and phrases. pointwise convergence, fractional Schrödinger equation.

This work was supported by NRF grant no. 2021R1A2B5B02001786, 2020R1I1A1A01072942 (C. Cho) 2017R1D1A1A02019547, and 2019R1A6A3A01092525 (H. Ko), South Korea.

*Corresponding author. 
necessary for the almost everywhere convergence. The sufficiency part of the convergence was shown by Du, Guth, and Li [10] when $d=2$ and by Du and Zhang [12] when $d \geq 3$ for a sharp range except for the endpoint (see $1,5,8,11,17,18,20,23$ for previous work).

For the fractional Schrödinger operator $(\alpha>1)$, Sjölin [21] proved that 1.2 holds if and only if $s \geq 1 / 4$ when $d=1$. He also obtained some positive results in higher dimensions: 1.2 is valid for $s \geq 1 / 2$ when $d=2$ and for $s>1 / 2$ when $d \geq 3$. Subsequently, this result was improved by Miao, Yang, and Zheng [19 to $s>3 / 8$ when $d=2$ and $s>s_{0}$ for some $s_{0}<1 / 2$ when $d \geq 3$. We extend the result for $d=2$.

Theorem 1.1. Let $\alpha>1$. Then, 1.2 holds for $f \in H^{s}\left(\mathbb{R}^{2}\right)$ whenever $s>1 / 3$.

The result in Theorem 1.1 extends to the solution of the linear dispersive equation

$$
i u_{t}-\Phi(D) u=0, \quad u(x, 0)=f(x)
$$

Here, $\Phi(D)$ is a multiplier operator defined on $\mathbb{R}^{2}$, where $\Phi$ is a smooth function except for the origin and satisfies the following property: for $\alpha>1$, there is a constant $C \geq 1$ such that $|\nabla \Phi(\xi)| \geq C^{-1}|\xi|^{\alpha-1}$ and $\left|\partial_{\xi}^{\gamma} \Phi(\xi)\right| \leq C|\xi|^{\alpha-|\gamma|}$ for any multi-indices $\gamma$. See Remark 3.11.

We denote by $B^{d}(x, r)$ a ball of radius $r$ centered at $x$ in $\mathbb{R}^{d}$. Theorem 1.1 follows from the maximal estimate.

Theorem 1.2. Let $\alpha>1$. Then, for $s>1 / 3$, there exists a constant $C>0$ such that

$$
\left\|U_{\alpha} f\right\|_{L_{x}^{3} L_{t}^{\infty}\left(B^{2}(0,1) \times[0,1]\right)} \leq C\|f\|_{H^{s}\left(\mathbb{R}^{2}\right)} .
$$

The proof of Theorem 1.2 is motivated by the argument used in [10 and proceeds by using polynomial partitioning to decompose $U_{\alpha} f$ into cells as well as transversal and tangential parts of a wall. The first two parts are easy to handle by induction, whereas the tangential term is much more complicated. To treat the tangential part, we need to prove refined Strichartz estimate for $U_{\alpha}$. We prove the estimate by using the decoupling inequality for elliptic parabola and induction on scales via rescaling. In contrast to the Schrödinger operator, $U_{\alpha} f(\alpha \neq 2)$ does not preserve the form after parabolic rescaling. To circumvent this issue, we consider a class of general elliptic functions as in [14]. Thus, we obtain the refined Strichartz estimates for a general class of operators (see Proposition 3.6).

Structure of the paper. The remainder of this paper is organized as follows. By applying polynomial partitioning, we reduce the problem to a problem of proving bilinear tangential estimate (Theorem 2.6). Section 3 establishes the linear refined Strichartz estimates (Proposition 3.6) and bilinear refined Strichartz estimates (Proposition 3.10). Accordingly, we prove Theorem 2.6. 
Notation. Throughout the paper, $\mathcal{F}(f)$ denotes the Fourier transform of $f$. Further, $A \lesssim B$ denotes $A \leq C B$ for some constant $C>0$ and $\# \mathcal{D}$ denotes the cardinality of a set $\mathcal{D}$.

\section{Proof of Theorem 1.2}

Let $\alpha>1$ and set $\mathbb{A}_{r}$ be the annulus given by

$$
\mathbb{A}_{r}:=\left\{\xi \in \mathbb{R}^{2}: 2^{-1} r \leq|\xi| \leq 2 r\right\}
$$

To prove Theorem 1.2 , by the Littlewood-Paley decomposition and the triangle inequality, it suffices to show that for any $\epsilon>0$, there is $C_{\epsilon}>0$ such that

$$
\left\|\sup _{0<t \leq 1}\left|U_{\alpha} f\right|\right\|_{L^{3}\left(B^{2}(0,1)\right)} \leq C_{\epsilon} R^{1 / 3+\epsilon}\|f\|_{2},
$$

provided that $\widehat{f}$ is supported on $\mathbb{A}_{R}$ for $R \geq 1$. By a parabolic rescaling $\xi \rightarrow R \xi$ and $(x, t) \rightarrow\left(R^{-1} x, R^{-\alpha} t\right)$, the estimate is reduced to showing that

$$
\left\|\sup _{0<t \leq R^{\alpha}}\left|U_{\alpha} f\right|\right\|_{L^{3}\left(B^{2}(0, R)\right)} \leq C_{\epsilon} R^{\epsilon}\|f\|_{2},
$$

whenever $\widehat{f}$ is supported on $\mathbb{A}_{1}$. Now we reduce the matter to showing 2.1) in which the supremum is taken over a smaller interval $[0, R]$ instead of $\left[0, R^{\alpha}\right]$. More precisely, to prove (2.1) it suffices to show that for any $\epsilon>0$, there exists a constant $C_{\epsilon}>0$ such that

$$
\left\|\sup _{0<t \leq R}\left|U_{\alpha} f\right|\right\|_{L^{3}\left(B^{2}(0, R)\right)} \leq C_{\epsilon} R^{\epsilon}\|f\|_{2}
$$

whenever $\widehat{f}$ is supported on $\mathbb{A}_{1}$. This reduction can be obtained by applying the time localization lemma in 19, Lemma 2.11] for the fractional Schrödinger operator (see also [16]). Alternatively, one may verify the lemma by using $T T^{*}$ argument as in 7, Lemma 2.1]. After finite decomposition, we may assume that $\widehat{f}$ is supported on a ball $B^{2}\left(\xi_{0}, r\right) \subset \mathbb{A}_{1}$. Hence, Theorem 1.2 is a consequence of the following. For simplicity, let $B_{R}=B^{2}(0, R) \times$ $[0, R]$.

Theorem 2.1. Let $p \geq 3$ and $R \geq 1$. Then, for any $\epsilon>0, q>\epsilon^{-4}$, and $r \leq 1$ such that $B\left(\xi_{0}, r\right) \subset \mathbb{A}_{1}$, there exists a constant $C_{\epsilon}>0$ such that

$$
\left\|U_{\alpha} f\right\|_{L_{x}^{p} L_{t}^{q}\left(B_{R}\right)} \leq C_{\epsilon} r^{\epsilon^{2}} R^{\epsilon}\|f\|_{2}
$$

whenever $\widehat{f}$ is supported on $B\left(\xi_{0}, r\right)$. 
Indeed, by the dominated convergence theorem, the estimate 2.2 implies that

$$
\left\|U_{\alpha} f\right\|_{L_{x}^{p} L_{t}^{\infty}\left(B_{R}\right)} \leq C_{\epsilon} R^{\epsilon}\|f\|_{2}
$$

for any $p>3$. By interpolating this with a trivial estimate $\left\|U_{\alpha} f\right\|_{L_{x}^{2} L_{t}^{\infty}\left(B_{R}\right)} \lesssim R^{1 / 2}\|f\|_{2}$, we have Theorem 1.2 .

We begin by stating a wave packet decomposition of $U_{\alpha} f$ (see, for example, [10,22]). For later use, we state the following for a more general operator $e^{i t \Phi} f$ defined by

$$
e^{i t \Phi} f(x, t)=\int e^{i(x \cdot \xi+t \Phi(\xi))} \widehat{f}(\xi) d \xi
$$

where $\Phi$ is smooth and the Hessian matrix of $\Phi$ is nondegenerate. Let $\psi$ be a smooth function such that $\widehat{\psi}$ is supported on $B^{2}(0,3 / 2)$ and $\sum_{k \in \mathbb{Z}^{2}}|\widehat{\psi}(\cdot-k)|^{2}=(2 \pi)^{-2}$ on $\mathbb{R}^{2}$. For $\delta>0$ and $(y, \nu) \in\left[R^{1 / 2} \mathbb{Z}^{2} \cap B^{2}(0, R)\right] \times\left[R^{-1 / 2} \mathbb{Z}^{2} \cap B^{2}(0,2)\right]$, we define a tube $T=T_{y, v}$ by

$$
T=\left\{(x, t) \in \mathbb{R}^{3}:|x-y+t \nabla \Phi(v)| \leq R^{1 / 2+\delta}, 0 \leq t \leq R\right\}
$$

and denote the direction of tube by $D(T)=(-\nabla \Phi(v), 1)$ and the set of all tubes $T$ by $\mathcal{T}$. We define $\psi_{T}=\psi_{T_{y, v}}$ by

$$
\widehat{\psi_{T}}(\xi)=e^{-i y \cdot \xi} R^{1 / 2} \widehat{\psi}\left(R^{1 / 2}(\xi-v)\right)
$$

so that $\sum_{T \in \mathcal{T}} \psi_{T}(x) \overline{\mathcal{F}\left(\psi_{T}\right)(\xi)}=(2 \pi)^{-2} e^{i x \cdot \xi}$ by the Poisson summation formula (see for example $[13]$ ).

Lemma 2.2. Let $\Phi, T$ and $\psi_{T}$ be as above. Suppose $\widehat{f}$ is supported on the ball $B^{2}(0,1)$. By setting $f_{T}=\left\langle f, \psi_{T}\right\rangle \psi_{T}$, we have

$$
f=\sum_{T \in \mathcal{T}} f_{T}
$$

such that

$$
\sum_{T \in \mathcal{T}}\left|\left\langle f, \psi_{T}\right\rangle\right|^{2} \lesssim\|f\|_{2}^{2}
$$

and for sufficiently large $N \geq 1$ and $(x, t) \in B^{3}(0, R)$,

$$
\left|e^{i t \Phi} \psi_{T}(x, t)\right| \lesssim R^{-1 / 2} \chi_{T}(x, t)+O\left(R^{-N}\right)\|f\|_{2} .
$$

Let $\epsilon>0$ and $0<r \leq 1 \leq R$. Suppose that the support of $\widehat{f}$ is contained in $B^{2}\left(\xi_{0}, r\right)$ for some $\xi_{0} \in \mathbb{A}_{1}$. The proof proceeds by induction on the size of $r$ and $R$. Note that (2.2) holds trivially for $R \sim 1$; hence, it suffices to consider $R \gg 1$. Furthermore, we only need to consider $r \geq R^{-1 / 2}$. In fact, if $r \leq R^{-10}$, then 2.2 follows trivially since 
$\left|U_{\alpha} f(x, t)\right| \leq r\|f\|_{2}$ by Hölder's inequality. On the other hand, if $R^{-10} \leq r \leq R^{-1 / 2}$, then all the wave packets have the same direction. Therefore, we apply Hölder's inequality and obtain

$$
\begin{aligned}
\left\|\sum_{T} U_{\alpha} f_{T}\right\|_{L_{x}^{p} L_{t}^{q}\left(B_{R}\right)} & \lesssim R^{-1 / 2+1 / q}\left\|\sum_{T}\left\langle f, \psi_{T}\right\rangle \chi_{T}\right\|_{L_{x}^{p} L_{t}^{\infty}\left(B_{R}\right)} \\
& \lesssim R^{-1 / 2+1 / q} \sum_{T}\left|\left\langle f, \psi_{T}\right\rangle\right|\left\|\sup _{T} \chi_{T}\right\|_{L_{x}^{p} L_{t}^{\infty}\left(B_{R}\right)} \\
& \lesssim R^{-1 / 2+1 / q} R^{(3 / 2+\delta) / p}\|f\|_{2} .
\end{aligned}
$$

For the last inequality, we use (2.3). Thus, 2.2 follows for $p \geq 3$ and sufficiently large $q>\epsilon^{-4}$ with small $\delta=\delta(\epsilon)$. Hereafter, we only consider $r \geq R^{-1 / 2}$. Now, we may assume that 2.2 holds if the radius of balls in physical space is less than $R / 2$ or the radius of balls in physical space is less than $R$ and that of balls in frequency space is less than $r / 2$. Then, it suffices to show 2.2 for $R \gg 1$ and $r \geq R^{-1 / 2}$.

Now, we reduce the matter to showing the bilinear tangential estimate (Theorem 2.6) by a standard argument using polynomial partitioning. Let us denote by $Z(P)$ the zero set of a polynomial $P$. We say that $P$ is a nonsingular polynomial if $\nabla P(z) \neq 0$ for all $z \in Z(P)$. Throughout this paper, we may assume that the polynomial $P$ is a product of nonsingular polynomials by the density argument (see [14]). We recall the polynomial partitioning in [10].

Theorem 2.3. Let $g \in L_{x}^{1} L_{t}^{s}\left(\mathbb{R}^{d+1}\right)$ be a nonzero function, $1 \leq s<\infty$, and $D>0$. Then, there exists a nonzero polynomial $P$ defined on $\mathbb{R}^{d+1}$ of degree $\leq D$, which is a product of distinct nonsingular polynomials, and there exists a collection of disjoint open sets $\left\{O_{i}\right\}_{i \in \mathcal{I}}$ such that $\# \mathcal{I} \sim D^{d+1}$ and

$$
\left(\mathbb{R}^{d} \times \mathbb{R}\right) \backslash Z(P)=\bigcup_{i \in \mathcal{I}} O_{i} .
$$

Moreover, there exists a constant $C_{1}$ independent of $i$ such that

$$
\|g\|_{L_{x}^{1} L_{t}^{s}\left(\mathbb{R}^{d+1}\right)} \leq C_{1} D^{d+1}\left\|\chi_{O_{i}} g\right\|_{L_{x}^{1} L_{t}^{s}\left(\mathbb{R}^{d+1}\right)}
$$

for each $i \in \mathcal{I}$.

By taking $s=q / p, D=R^{\epsilon^{4}}$, and $g=\chi_{B_{R}}\left|U_{\alpha} f\right|^{p}$, and applying Theorem 2.3, we have

$$
\left\|U_{\alpha} f\right\|_{L_{x}^{p} L_{t}^{q}\left(B_{R}\right)}^{p} \leq C_{1} D^{3}\left\|\chi_{O_{i}} U_{\alpha} f\right\|_{L_{x}^{p} L_{t}^{q}\left(B_{R}\right)}^{p} .
$$

We denote by $W$ a wall that is an $R^{1 / 2+\delta}$-neighborhood of $Z(P)$ and a cell $\widetilde{O}_{i}=O_{i} \backslash W$. It is clear that

$$
\left\|U_{\alpha} f\right\|_{L_{x}^{p} L_{t}^{q}\left(B_{R}\right)}^{p}=\sum_{i \in \mathcal{I}}\left\|\chi_{\widetilde{O}_{i}} U_{\alpha} f\right\|_{L_{x}^{p} L_{t}^{q}\left(B_{R}\right)}^{p}+\left\|\chi_{W} U_{\alpha} f\right\|_{L_{x}^{p} L_{t}^{q}\left(B_{R}\right)}^{p}
$$


If the terms $\sum_{i \in \mathcal{I}}\left\|\chi_{\widetilde{O}_{i}} U_{\alpha} f\right\|_{L_{x}^{p} L_{t}^{q}\left(B_{R}\right)}^{p}$ dominate the other in the above-mentioned equation, the estimate is easy to handle. Hence, we consider this case first.

Cellular part. Let us consider a subcollection $\widetilde{\mathcal{I}}$ of an index set $\mathcal{I}$ such that

$$
\widetilde{\mathcal{I}}=\left\{i \in \mathcal{I}:\left\|U_{\alpha} f\right\|_{L_{x}^{p} L_{t}^{q}\left(B_{R}\right)}^{p} \leq 2 C_{1} D^{3}\left\|\chi_{\widetilde{O}_{i}} U_{\alpha} f\right\|_{L_{x}^{p} L_{t}^{q}\left(B_{R}\right)}^{p}\right\}
$$

where the constant $C_{1}$ is given by 2.5 . To treat the case in which the cellular part dominates the walls, we may assume that $\widetilde{\mathcal{I}}=\mathcal{I}$. For each $i \in \mathcal{I}$, we set

$$
f_{i}=\sum_{T: T \cap \widetilde{O}_{i} \neq \emptyset} f_{T}
$$

By Lemma 2.2, if $(x, t) \in \widetilde{O}_{i}$, then $\left|U_{\alpha} f(x, t)\right| \leq\left|U_{\alpha} f_{i}(x, t)\right|+O\left(R^{-N}\right)\|f\|_{2}$ for sufficiently large $N$. Since each tube $T$ intersects at most $(D+1)$ cells $O_{i}$, we have

$$
\sum_{i \in \mathcal{I}}\left\|f_{i}\right\|_{2}^{2}=\sum_{\substack{i \in \mathcal{I}, T \in \mathcal{T} \\ T \cap \widetilde{O}_{i} \neq \emptyset}}\left\|f_{T}\right\|_{2}^{2} \lesssim D\|f\|_{2}^{2}
$$

Since $\# \mathcal{I} \sim D^{3}$, by pigeonholing, there exists an index $i_{\circ} \in \mathcal{I}$ such that $\left\|f_{i_{\circ}}\right\|_{2}^{2} \lesssim D^{-2}\|f\|_{2}^{2}$. We cover $B_{R}$ by $\left\{B_{R / 2}^{\prime}\right\}$, which are translations of $B_{R / 2}$, and obtain

$$
\left\|U_{\alpha} f\right\|_{L_{x}^{p} L_{t}^{q}\left(B_{R}\right)}^{p} \leq 2 C_{1} D^{3} \sum_{B_{R / 2}^{\prime}}\left\|\sum_{T: T \cap \widetilde{O}_{i_{\circ}} \neq \emptyset} U_{\alpha} f_{T}\right\|_{L_{x}^{p} L_{t}^{q}\left(B_{R / 2}^{\prime}\right)}^{p}+O\left(R^{-N}\right)\|f\|_{2}^{p} .
$$

By applying the induction hypothesis, it follows that

$$
\begin{aligned}
\left\|U_{\alpha} f\right\|_{L_{x}^{p} L_{t}^{q}\left(B_{R}\right)}^{p} & \leq C D^{3}\left[C_{\epsilon} r^{\epsilon^{2}}(R / 2)^{\epsilon}\left\|f_{i_{\circ}}\right\|_{2}\right]^{p}+O\left(R^{-N}\right)\|f\|_{2}^{p} \\
& \leq 2 C 2^{-p \epsilon} D^{3}\left[C_{\epsilon} r^{\epsilon^{2}} R^{\epsilon} D^{-1}\|f\|_{2}\right]^{p} .
\end{aligned}
$$

Since $p>3$ and $D=R^{\epsilon^{4}}$, we see that $2 C 2^{-p \epsilon} D^{3-p} \leq 2^{-p}$ for sufficiently large $R$. Therefore, we get 2.2 when the cell part dominates.

Now, we consider the opposite case in which the wall part dominates. To this end, we present some definitions. We denote by $T_{z}(Z(P))$ the tangent plane of $Z(P)$ at a fixed point $z$. Let us partition $B_{R}$ into balls $B_{j}$ of radius $R^{1-\delta}$. We say that a tube $T$ is tangent to the wall $W$ in $B_{j}$ if $T$ intersects $B_{j}$ and $W$ and satisfies

$$
\text { Angle }\left(D(T), T_{z}(Z(P))\right) \leq R^{-1 / 2+2 \delta}
$$

for any nonsingular point $z \in Z(P) \cap 10 T \cap 2 B_{j}$. Otherwise, we say that $T$ is transversal to the wall $W$ in $B_{j}$. Let $\mathcal{T}_{j \text {,tang }}$ be the collection of all tubes $T \in \mathcal{T}$ such that $T$ is tangent 
to the wall in $B_{j}$ and let $\mathcal{T}_{j \text {,trans }}$ be the collection of tubes such that $T$ is transversal. We also set

$$
f_{j, \text { tang }}=\sum_{T \in \mathcal{T}_{j, \text { tang }}} f_{T} \text { and } f_{j, \text { trans }}=\sum_{T \in \mathcal{T}_{j, \text { trans }}} f_{T} .
$$

For a given $\delta^{\prime}>0$, we say that a tube $T$ is $R^{-1 / 2+\delta^{\prime}}$-tangent to $Z$ if it satisfies

$$
T \subset N_{R^{1 / 2+\delta^{\prime}}} Z \cap B_{R}, \quad \text { Angle }\left(D(T), T_{z} Z(P)\right) \leq R^{-1 / 2+\delta^{\prime}}
$$

for all nonsingular points $z \in N_{2 R^{1 / 2+\delta^{\prime}}}(T) \cap 2 B_{R} \cap Z$. The collection of tubes that are $R^{-1 / 2+\delta^{\prime}}$-tangent to $Z$ is denoted by $T_{Z}\left(R^{-1 / 2+\delta^{\prime}}\right)$. We say that $f$ is concentrated on wave packets from $T_{Z}\left(R^{-1 / 2+\delta^{\prime}}\right)$ if

$$
\sum_{T \notin T_{Z}\left(R^{-1 / 2+\delta^{\prime}}\right)}\left\|f_{T}\right\|_{2}=O\left(R^{-N}\right)\|f\|_{2}
$$

holds for sufficiently large $N>0$.

Wall part. Now we consider the case $\mathcal{I} \neq \tilde{\mathcal{I}}$; hence, we can choose $i_{\circ} \in \mathcal{I} \backslash \widetilde{\mathcal{I}}$. From 2.5,

$$
\left\|U_{\alpha} f\right\|_{L_{x}^{p} L_{t}^{q}\left(B_{R}\right)}^{p} \leq C_{1} D^{3}\left\|\chi_{\widetilde{O}_{i_{\circ}}} U_{\alpha} f\right\|_{L_{x}^{p} L_{t}^{q}\left(B_{R}\right)}^{p}+C_{1} D^{3}\left\|\chi_{O_{i_{\circ}} \cap W} U_{\alpha} f\right\|_{L_{x}^{p} L_{t}^{q}\left(B_{R}\right)}^{p} .
$$

Since $i_{\circ} \in \mathcal{I} \backslash \widetilde{\mathcal{I}}$, we have $C_{1} D^{3}\left\|\chi_{\widetilde{O}_{i_{\circ}}} U_{\alpha} f\right\|_{L_{x}^{p} L_{t}^{q}\left(B_{R}\right)}^{p} \leq 2^{-1}\left\|U_{\alpha} f\right\|_{L_{x}^{p} L_{t}^{q}\left(B_{R}\right)}^{p}$. Therefore,

$$
\left\|U_{\alpha} f\right\|_{L_{x}^{p} L_{t}^{q}\left(B_{R}\right)}^{p} \leq 2 C_{1} D^{3}\left\|\chi_{W} U_{\alpha} f\right\|_{L_{x}^{p} L_{t}^{q}\left(B_{R}\right)}^{p} .
$$

Thus, it suffices to consider the wave packets concentrated on the wall.

Recalling that supp $\widehat{f} \subset B^{2}\left(\xi_{0}, r\right)$, for $1 \ll K \ll R^{\epsilon}$, we cover $B^{2}\left(\xi_{0}, r\right)$ by boundedly overlapping collection of balls $\omega$ of radius $K^{-1} r$ and let $f=\sum_{\omega} f_{\omega}$, where $\widehat{f_{\omega}}$ is supported on $\omega$. For each fixed $B_{j}$, we set

$$
f_{\omega, j, \operatorname{tang}}=\left(f_{\omega}\right)_{j, \operatorname{tang}}, \quad f_{\omega, j, \operatorname{trans}}=\left(f_{\omega}\right)_{j, \operatorname{trans}}
$$

We define a bilinear tangential operator by

$$
\operatorname{Bil}\left(U_{\alpha} f_{j, \text { tang }}\right)(x, t)=\sum_{\operatorname{dist}\left(\omega_{1}, \omega_{2}\right) \geq K^{-1} r}\left|U_{\alpha} f_{\omega_{1}, j, \operatorname{tang}}(x, t)\right|^{1 / 2}\left|U_{\alpha} f_{\omega_{2}, j, \operatorname{tang}}(x, t)\right|^{1 / 2} .
$$

We set

$$
B=\left\{(x, t) \in B_{R}: K^{\epsilon^{3}} \max _{\omega}\left|U_{\alpha} f_{\omega}(x, t)\right| \leq\left|U_{\alpha} f(x, t)\right|\right\}
$$

and for $(x, t) \in W \cap B$,

$$
\Omega=\left\{\omega:\left|U_{\alpha} f_{\omega, j, \operatorname{tang}}(x, t)\right| \leq K^{-4}\left|U_{\alpha} f(x, t)\right|\right\} .
$$


By fixing $B_{j}$ and $(x, t) \in B_{j} \cap W \cap B$, we first consider the case in which all balls $\omega$ in $\Omega^{\mathrm{c}}$ are adjacent. Hence, $\# \Omega^{\mathrm{c}} \lesssim 1$, and it follows that $\sum_{\omega \in \Omega^{\mathrm{c}}}\left|U_{\alpha} f_{\omega}(x, t)\right| \leq \frac{1}{2}\left|U_{\alpha} f(x, t)\right|$ by (2.7). Thus, we have $\frac{1}{2}\left|U_{\alpha} f(x, t)\right| \leq\left|\sum_{\omega \in \Omega} U_{\alpha} f_{\omega}(x, t)\right|$. Then,

$$
\frac{1}{2}\left|U_{\alpha} f(x, t)\right| \leq\left|\sum_{\omega \in \Omega} U_{\alpha} f_{\omega, j, \operatorname{trans}}(x, t)\right|+\left|\sum_{\omega \in \Omega} U_{\alpha} f_{\omega, j, \operatorname{tang}}(x, t)\right|+O\left(R^{-N}\right)\|f\|_{2} .
$$

Since the total number of $\omega$ is $\leq 10 K^{2}$, we get

$$
\frac{1}{2}\left|U_{\alpha} f(x, t)\right| \lesssim\left|\sum_{\omega \in \Omega} U_{\alpha} f_{\omega, j, \operatorname{trans}}(x, t)\right|+K^{-2}\left|U_{\alpha} f(x, t)\right|+O\left(R^{-N}\right)\|f\|_{2} .
$$

Otherwise, for $(x, t) \in B_{j} \cap W \cap B$, there are $\omega_{1}, \omega_{2} \in \Omega^{\mathrm{c}} \operatorname{such}$ that $\operatorname{dist}\left(\omega_{1}, \omega_{2}\right) \gtrsim K^{-1} r$. Then, by the definition of $\Omega,\left|U_{\alpha} f(x, t)\right| \leq K^{4} \operatorname{Bil}\left(U_{\alpha} f_{j, \operatorname{tang}}\right)(x, t)$. Therefore, we have the following.

Lemma 2.4. For each point $(x, t) \in W \cap B_{R}$, there exists a collection $\Omega$ of balls $\omega$ of radius $K^{-1} r$ such that

$$
\begin{aligned}
\left|\chi_{W} U_{\alpha} f(x, t)\right|^{p} \lesssim & \left|\chi_{W \cap B^{c}} U_{\alpha} f(x, t)\right|^{p}+\sum_{j}\left|\sum_{\omega \in \Omega} \chi_{W \cap B_{j}} U_{\alpha} f_{\omega, j, \text { trans }}(x, t)\right|^{p} \\
& +\sum_{j} K^{4 p}\left|\chi_{W \cap B_{j}} \operatorname{Bil}\left(U_{\alpha} f_{j, \text { tang }}\right)(x, t)\right|^{p}+O\left(R^{-N}\right)\|f\|_{2}^{p} .
\end{aligned}
$$

By Lemma 2.4, we have

$$
\begin{aligned}
\left\|U_{\alpha} f\right\|_{L_{x}^{p} L_{t}^{q}\left(W \cap B_{R}\right)}^{p} \lesssim & \left\|U_{\alpha} f\right\|_{L_{x}^{p} L_{t}^{q}\left(W \cap B^{c}\right)}^{p}+\sum_{j}\left\|\sum_{\omega \in \Omega} U_{\alpha} f_{\omega, j, \operatorname{trans}}\right\|_{L_{x}^{p} L_{t}^{q}\left(W \cap B_{j}\right)}^{p} \\
& +\sum_{j}\left\|K^{4} \operatorname{Bil}\left(U_{\alpha} f_{j, \operatorname{tang}}\right)\right\|_{L_{x}^{p} L_{t}^{q}\left(W \cap B_{j}\right)}^{p}+O\left(R^{-N}\right)\|f\|_{2}^{p} .
\end{aligned}
$$

From (2.7), the first term on the right-hand side of 2.8 is bounded by

$$
\left\|U_{\alpha} f\right\|_{L_{x}^{p} L_{t}^{q}\left(W \cap B^{c}\right)}^{p} \leq K^{\epsilon^{3} p} \sum_{\omega}\left\|U_{\alpha} f_{\omega}\right\|_{L_{x}^{p} L_{t}^{q}\left(B_{R}\right)}^{p} .
$$

By applying the induction hypothesis 2.2 to the right-hand side, we have

$$
\left\|U_{\alpha} f\right\|_{L_{x}^{p} L_{t}^{q}\left(W \cap B^{c}\right)}^{p} \leq K^{\epsilon^{3} p} \sum_{\omega}\left[C_{\epsilon}\left(K^{-1} r\right)^{\epsilon^{2}} R^{\epsilon}\left\|f_{\omega}\right\|_{2}\right]^{p} \leq 10 K^{\left(\epsilon^{3}-\epsilon^{2}\right) p}\left[C_{\epsilon} r^{\epsilon^{2}} R^{\epsilon}\|f\|_{2}\right]^{p}
$$

Since $K \ll R^{\epsilon}$ and $R \gg 1$, we have $10 K^{\epsilon^{3}-\epsilon^{2}} \leq 1 / 6$. This completes the induction step for the first term on the right-hand side of (2.8). In the remainder of this section, we treat the second and third terms on the right-hand side of 2.8. 
Transversal case. Now, we deal with the transversal term in (2.8). In [14, it was shown that for each tube $T \in \mathcal{T}$,

$$
\#\left\{j: T \in \mathcal{T}_{j, \text { trans }}\right\} \leq R^{O\left(\epsilon^{4}\right)} .
$$

Since $\# \Omega \leq 2^{10 K^{2}}$, the second term on the right-hand side of $(2.8)$ is controlled by

$$
\left.\sum_{j}\left\|\max _{\Omega}\left|\sum_{\omega \in \Omega} U_{\alpha} f_{\omega, j, \operatorname{trans}}\right|\right\|\right|_{L_{x}^{p} L_{t}^{q}\left(W \cap B_{j}\right)} ^{p} \leq \sum_{j} 2^{10 K^{2}}\left\|\sum_{\omega \in \Omega} U_{\alpha} f_{\omega, j, \operatorname{trans}}\right\|_{L_{x}^{p} L_{t}^{q}\left(B_{j}\right)}^{p} .
$$

By applying the induction hypothesis to a ball $B_{j}$ of radius $R^{1-\delta}$, the right-hand side of (2.10) is bounded by $\sum_{j} 2^{10 K^{2}}\left[C_{\epsilon} r^{\epsilon^{2}} R^{(1-\delta) \epsilon}\left\|f_{j, \text { trans }}\right\|_{2}\right]^{p}$. Therefore, by applying (2.9), we get

$$
\sum_{j}\left\|\max _{\Omega} \mid \sum_{\omega \in \Omega} U_{\alpha} f_{\omega, j, \operatorname{trans}}\right\| \|_{L_{x}^{p} L_{t}^{q}\left(W \cap B_{j}\right)}^{p} \leq R^{O\left(\epsilon^{4}\right)} 2^{10 K^{2}} R^{-\delta \epsilon p}\left[C_{\epsilon} r^{\epsilon^{2}} R^{\epsilon}\|f\|_{2}\right]^{p} .
$$

Since $K \ll R^{\epsilon}$, we take $\delta=\epsilon^{2}$ and obtain $2^{10 K^{2}} R^{O\left(\epsilon^{4}\right)-\epsilon^{3} p} \leq 1 / 6$ for sufficiently large $R>0$. Therefore, the induction closes for the transversal term.

Bilinear tangential case. To estimate the third term on the right-hand side of (2.8), it remains to prove the following bilinear maximal estimates.

Theorem 2.5. For any $\epsilon>0$ and $p>3$, there exists $C_{\epsilon}>0$ such that

$$
\left(\int_{B_{R} t:(x, t) \in W \cap B_{j}}\left|\operatorname{Bil}\left(U_{\alpha} f_{j, \text { tang }}\right)(x, t)\right|^{p} d x\right)^{1 / p} \leq C_{\epsilon} R^{\epsilon / 2}\|f\|_{2} .
$$

Indeed, assuming Theorem 2.5, by Hölder's inequality, it follows that for $q>\epsilon^{-4}$,

$$
\begin{aligned}
\sum_{j} K^{4 p}\left\|\operatorname{Bil}\left(U_{\alpha} f_{j, \text { tang }}\right)\right\|_{L_{x}^{p} L_{t}^{q}\left(W \cap B_{j}\right)}^{p} & \leq \sum_{j} K^{4 p} R^{\epsilon^{4} p}\left\|\operatorname{Bil}\left(U_{\alpha} f_{j, \text { tang }}\right)\right\|_{L_{x}^{p} L_{t}^{\infty}\left(W \cap B_{j}\right)}^{p} \\
& \leq R^{3 \delta} K^{4 p} R^{\epsilon^{4} p}\left[C_{\epsilon} R^{\epsilon / 2}\|f\|_{2}\right]^{p}
\end{aligned}
$$

since the number of $j$ is $\lesssim R^{3 \delta}$. Because $\delta=\epsilon^{2}, K \ll R^{\epsilon}$ and $r \geq R^{-1 / 2}$, we obtain $K^{4} R^{\epsilon^{4}+\epsilon / 2+3 \delta / p} \leq \frac{1}{6} R^{\epsilon} r^{\epsilon^{2}}$. This completes the proof of Theorem 2.1.

To prove Theorem 2.5, we show the following maximal estimate.

Theorem 2.6. Let $0<r \leq 1, \xi_{0} \in \mathbb{A}_{1}$, and $K=K(\epsilon)$ be a sufficiently large constant. Suppose that the supports of $\widehat{f}$ and $\widehat{g}$ are contained in $B^{2}\left(\xi_{0}, r\right)$ and separated by $K^{-1} r$. If $f, g$ are concentrated on the wave packets from $T_{Z}\left(R^{-1 / 2+\delta^{\prime}}\right)$ with $\delta^{\prime} \leq 100 \delta$, then there exist constants $c$ and $C$ such that

$$
\left\|\left|U_{\alpha} f\right|^{1 / 2}\left|U_{\alpha} g\right|^{1 / 2}\right\|_{L_{x}^{3} L_{t}^{\infty}\left(B_{R}\right)} \leq C R^{c \delta^{\prime}}\|f\|_{2}^{1 / 2}\|g\|_{2}^{1 / 2} .
$$


In Theorem 2.5, we are concerned with the function $f_{j \text {,tang }}$ defined on a smaller ball $B_{j}$ of radius $R_{1}=R^{1-\delta}$. We can easily see that the wave packets of $f_{j \text {,tang }}$ on the ball $B_{j}$ are concentrated in $T_{Z}\left(R_{1}^{-1 / 2+\delta^{\prime}}\right.$ ) for some $\delta^{\prime} \leq 100 \delta$ (see |15|), and we omit the details. Since $R_{1}^{\delta^{\prime}} \leq R^{c \delta}$, we obtain the desired bound in Theorem 2.5 from the estimate 2.11.

\section{Proof of Theorem 2.6}

In this section, we prove Theorem 2.6 by considering linear and bilinear refined Strichartz estimates (Propositions 3.6 and 3.10 respectively), which are variants of the estimates presented in [10 for a class of elliptic functions. We begin by defining a class of elliptic phase functions.

\subsection{Class of elliptic functions}

We consider the class of phase functions that are small perturbations of $\phi_{0}(\xi)=|\xi|^{2} / 2$.

Definition 3.1. Let $0<\epsilon_{0} \ll 1, \rho>0$, and $n \geq 10^{3}$ be a positive integer. We define a class of normalized phase functions by

$$
\mathcal{P}\left(\epsilon_{0}, n\right)=\left\{\phi \in \mathcal{C}^{n}\left(B^{2}(0,2)\right):\left\|\phi-\phi_{0}\right\|_{\mathcal{C}^{n}\left(B^{2}(0,2)\right)} \leq \epsilon_{0}\right\}
$$

Let $\phi \in \mathcal{P}\left(\epsilon_{0}, n\right), \xi_{0} \in \mathbb{A}_{1}$, and $\mathrm{H} \phi\left(\xi_{0}\right)$ be the Hessian matrix of $\phi$ at $\xi=\xi_{0}$. Then, $\mathrm{H} \phi$ is positive definite on $B^{2}(0,2)$ and $\mathrm{H} \phi\left(\xi_{0}\right)=\mathrm{T}^{-1} \mathrm{DT}$, where $\mathrm{D}$ is a diagonal matrix with eigenvalues $\lambda_{1}>0$ and $\lambda_{2}>0$, and $\mathrm{T}$ is a symmetric matrix. If we set $\mathrm{H}_{\xi_{0}}:=\sqrt{\mathrm{H} \phi\left(\xi_{0}\right)}$, then $\mathrm{H}_{\xi_{0}}=\mathrm{T}^{-1} \mathrm{D}^{1 / 2} \mathrm{~T}$ with $\mathrm{D}^{1 / 2}=\left(\sqrt{\lambda_{1}} e_{1}, \sqrt{\lambda_{2}} e_{2}\right)$. We denote the normalization of $\phi$ by

$$
\phi_{\xi_{0}}^{\rho}(\xi)=\rho^{-2}\left(\phi\left(\rho \mathrm{H}_{\xi_{0}}^{-1} \xi+\xi_{0}\right)-\phi\left(\xi_{0}\right)-\rho \nabla \phi\left(\xi_{0}\right) \cdot \mathrm{H}_{\xi_{0}}^{-1} \xi\right)
$$

Then, we observe the following, which plays an important role in the induction argument. We denote by int $\mathbb{A}_{r}$ the interior of $\mathbb{A}_{r}$.

Lemma 3.2. Let $\epsilon_{0}>0$ and $\xi_{0} \in \operatorname{int} \mathbb{A}_{1}$. Suppose that $\phi \in \mathcal{C}^{n}\left(\mathbb{A}_{1}\right)$ and the Hessian matrix of $\phi$ is positive definite. Then, there exists a constant $\rho_{0}>0$ such that $\phi_{\xi_{0}}^{\rho} \in \mathcal{P}\left(\epsilon_{0}, n\right)$ whenever $\rho \leq \rho_{0}$. Moreover, if $\phi \in \mathcal{P}\left(\epsilon_{0}, n\right)$, then for sufficiently small $\epsilon_{0}>0$, there exists a constant $\rho_{1}$ such that $\phi_{\xi_{0}}^{\rho} \in \mathcal{P}\left(\epsilon_{0}, n\right)$ whenever $\rho \leq \rho_{1}$.

Proof. By (3.1) and Taylor's expansion, we may write

$$
\phi_{\xi_{0}}^{\rho}(\xi)=\frac{|\xi|^{2}}{2}+\mathcal{E}\left(\xi, \xi_{0}, \rho\right)
$$

where $\left\|\mathcal{E}\left(\cdot, \xi_{0}, \rho\right)\right\|_{\mathcal{C}^{n}\left(\mathbb{A}_{1}\right)}=O\left(\rho\left|\mathrm{H}_{\xi_{0}}^{-1} \xi\right|^{3}\right)$. Thus, we can take $\rho_{0}$ such that $\left\|\phi_{\xi_{0}}^{\rho}-\phi_{0}\right\|_{\mathcal{C}^{n}\left(\mathbb{A}_{1}\right)} \leq$ $C \rho \leq \epsilon_{0}$ holds for any $\rho \leq \rho_{0}$. Similarly, if $\phi \in \mathcal{P}\left(\epsilon_{0}, n\right)$ and $\xi_{0} \in B^{2}(0,1)$, then we can take $\rho_{1}>0$ such that $\phi_{\xi_{0}}^{\rho} \in \mathcal{P}\left(\epsilon_{0}, n\right)$ whenever $\rho \leq \rho_{1}$. 
Suppose that $\phi \in \mathcal{C}^{n}\left(\mathbb{A}_{1}\right)$ and the Hessian matrix of $\phi$ is positive definite. For a given small $\epsilon_{0}>0$, by partitioning $B^{2}(0,1)$ into smaller balls of radius $\rho_{0}$ and applying Lemma $3.2, \phi_{\xi_{0}}^{\rho} \in \mathcal{P}\left(\epsilon_{0}, n\right)$ for any $\rho \leq \rho_{0}$. Therefore, hereafter, we may fix $n \geq 10^{3}$ and simply denote $\mathcal{P}\left(\epsilon_{0}, n\right)$ by $\mathcal{P}\left(\epsilon_{0}\right)$. To prove Theorem 2.6, it suffices to consider $\phi \in \mathcal{P}\left(\epsilon_{0}\right)$.

\subsection{Parabolic rescaling}

We define a linear map $\mathcal{A}_{\xi_{0}}^{\rho}: \mathbb{R}^{3} \rightarrow \mathbb{R}^{3}$ by

$$
\left(\mathcal{A}_{\xi_{0}}^{\rho}\right)^{-1}(x, t)=\left(\rho^{-1} \mathrm{H}_{\xi_{0}}^{t} x-\rho^{-2} t \nabla \phi\left(\xi_{0}\right), \rho^{-2} t\right) .
$$

Lemma 3.3. Let $\epsilon_{0}>0$ be sufficiently small, $\xi_{0} \in \operatorname{int} \mathbb{A}_{1}$, and $\phi \in \mathcal{P}\left(\epsilon_{0}\right)$. Suppose that $\widehat{f}$ is supported on a ball $B^{2}\left(\xi_{0}, \rho\right)$ for $\rho \leq \rho_{1}$, where $\rho_{1}$ is given in Lemma 3.2. Then, there exist $\widetilde{f}, \widetilde{T}$, and a constant $C=C\left(\xi_{0}, \rho\right)$ such that

$$
\left\|e^{i t \phi} f\right\|_{L^{q}(T)}=C \rho^{1-4 / q} \| e^{i t \phi_{\xi_{0}}^{\rho} \widetilde{f} \|_{L^{q}(\widetilde{T})},}
$$

where $\mathcal{F}(\widetilde{f})$ is supported on $B^{2}(0,1)$ such that $\|\widetilde{f}\|_{2}=\|f\|_{2}$, and

$$
\widetilde{T}=\left\{(x, t):\left(\mathcal{A}_{\xi_{0}}^{\rho}\right)^{-1}(x, t) \in T\right\}
$$

Remark 3.4. Let $\phi \in \mathcal{P}\left(\epsilon_{0}\right)$. Suppose that $T$ is a tube of dimensions $\rho^{-1} M \times \rho^{-1} M \times \rho^{-2} M$ centered at the origin with its long axis parallel to $\left(-\nabla \phi\left(\xi_{0}\right), 1\right)$, i.e.,

$$
T=\left\{(x, t):\left|x+t \nabla \phi\left(\xi_{0}\right)\right| \leq \rho^{-1} M,|t| \leq \rho^{-2} M\right\} .
$$

Then, $\widetilde{T}=\left\{(x, t):\left|\rho^{-1} \mathrm{H}_{\xi_{0}} x\right| \leq \rho^{-1} M,\left|\rho^{-2} t\right| \leq \rho^{-2} M\right\}$ and $\widetilde{T}$ is contained a cube of side length $C M$ for some $C=C\left(\epsilon_{0}\right)$.

Proof of Lemma 3.3. Let $|\mathrm{H}|$ denote the determinant of a matrix H. By change of variables $\xi \rightarrow \rho \mathrm{H}_{\xi_{0}}^{-1} \xi+\xi_{0}$, we have

$$
\left|e^{i t \phi} f(x)\right|=\left.\rho\left|\mathrm{H}_{\xi_{0}}^{-1}\right|^{1 / 2}\left|\int e^{i(x, t) \cdot\left(\rho \mathrm{H}_{\xi_{0}}^{-1} \xi, \phi\left(\rho \mathrm{H}_{\xi_{0}}^{-1} \xi+\xi_{0}\right)\right)} \rho\right| \mathrm{H}_{\xi_{0}}^{-1}\right|^{1 / 2} \widehat{f}\left(\rho \mathrm{H}_{\xi_{0}}^{-1} \xi+\xi_{0}\right) d \xi \mid .
$$

We define $\tilde{f}$ by

$$
\mathcal{F} \widetilde{f}(\xi)=\rho\left|\mathrm{H}_{\xi_{0}}^{-1}\right|^{1 / 2} \widehat{f}\left(\rho \mathrm{H}_{\xi_{0}}^{-1} \xi+\xi_{0}\right)
$$

Then, $\tilde{f}$ has a Fourier support on $B^{2}(0,1)$ and $\|\widetilde{f}\|_{2}=\|f\|_{2}$. By the definition of $\phi_{\xi_{0}}^{\rho}$ (see (3.1), we note that

$$
\left(\rho^{-1} \mathrm{H}_{\xi_{0}}^{t} x-\rho^{-2} t \nabla \phi\left(\xi_{0}\right)\right) \cdot \rho \mathrm{H}_{\xi_{0}}^{-1} \xi+\rho^{-2} t\left(\phi\left(\rho \mathrm{H}_{\xi_{0}}^{-1} \xi+\xi_{0}\right)-\phi\left(\xi_{0}\right)\right)=x \cdot \xi+t \phi_{\xi_{0}}^{\rho}(\xi) .
$$

Thus, by change of variables $x \rightarrow \rho^{-1} \mathrm{H}_{\xi_{0}}^{t} x-t \nabla \phi\left(\xi_{0}\right)$ and $t \rightarrow \rho^{-2} t$ (i.e., $(x, t) \rightarrow$ $\left.\left(\mathcal{A}_{\xi_{0}}^{\rho}\right)^{-1}(x, t)\right)$, the desired bound 3.3 follows by taking $C=\left|\mathrm{H}_{\xi_{0}}\right|^{1 / q-1 / 2}$. 
Now, we observe that the condition 2.6 is preserved after parabolic rescaling.

Lemma 3.5. Let $0<\rho \leq \rho_{0}$ and $\xi_{0} \in \operatorname{int} \mathbb{A}_{1}$. Suppose that $f$ is concentrated on the wave packets from $T_{Z}\left(R^{-1 / 2+\delta^{\prime}}\right)$ associated with $\phi$ for some $Z=Z(P)$. If $\tilde{f}$ is given by (3.4), then $\widetilde{f}$ is concentrated on the wave packets from $T_{Z^{\prime}}\left(\rho^{-1} R^{-1 / 2+\delta^{\prime}}\right)$ associated with $\phi_{\xi_{0}}^{\rho}$ and $Z^{\prime}=Z^{\prime}(\widetilde{P})$, where $\widetilde{P}=P \circ\left(\mathcal{A}_{\xi_{0}}^{\rho}\right)^{-1}$.

Proof. It suffices to show that

$$
\left|\left(-\nabla_{\xi^{\prime}} \phi_{\xi_{0}}^{\rho}\left(\xi^{\prime}\right), 1\right) \cdot \nabla_{x^{\prime}, t^{\prime}} \widetilde{P}\right| /\left|\nabla_{x^{\prime}, t^{\prime}} \widetilde{P}\right| \lesssim \rho^{-1} R^{-1 / 2+\delta^{\prime}},
$$

where $\xi^{\prime}=\rho^{-1} \mathrm{H}_{\xi_{0}}\left(\xi-\xi_{0}\right)$ and $\left(x^{\prime}, t^{\prime}\right)=\mathcal{A}_{\xi_{0}}^{\rho}(x, t)$. Since $\widetilde{P}\left(x^{\prime}, t^{\prime}\right)=P\left(\rho^{-1} \mathrm{H}_{\xi_{0}}^{t} x^{\prime}-\right.$ $\left.\rho^{-2} t^{\prime} \nabla \phi\left(\xi_{0}\right), \rho^{-2} t^{\prime}\right)$, we have

$$
\nabla_{x^{\prime}} \widetilde{P}=\rho^{-1} \mathrm{H}_{\xi_{0}} \nabla_{x} P \quad \text { and } \quad \partial_{t^{\prime}} \widetilde{P}=\rho^{-2}\left(\partial_{t} P-\nabla_{x} P \cdot \nabla \phi\left(\xi_{0}\right)\right) .
$$

Combining this with $\nabla_{\xi^{\prime}} \phi_{\xi_{0}}^{\rho}\left(\xi^{\prime}\right)=\rho^{-1} \mathrm{H}_{\xi_{0}}^{-t}\left(\nabla \phi(\xi)-\nabla \phi\left(\xi_{0}\right)\right)$, we get

$$
\left(-\nabla_{\xi^{\prime}} \phi_{\xi_{0}}^{\rho}\left(\xi^{\prime}\right), 1\right) \cdot\left(\nabla_{x^{\prime}} \widetilde{P}, \partial_{t^{\prime}} \widetilde{P}\right)=\rho^{-2}(-\nabla \phi(\xi), 1) \cdot\left(\nabla_{x} P, \partial_{t} P\right) .
$$

On the other hand, from (3.6), we can easily deduce that $\left|\nabla_{x^{\prime}, t^{\prime}} \widetilde{P}\right| \gtrsim \rho^{-1}\left|\nabla_{x, t} P\right|$ by considering the cases $\left|\partial_{t} P\right| \geq 2\left|\nabla_{x} P \cdot \nabla \phi\left(\xi_{0}\right)\right|$ and $\left|\partial_{t} P\right| \leq 2\left|\nabla_{x} P \cdot \nabla \phi\left(\xi_{0}\right)\right|$ separately. By the assumption $\left|(-\nabla \phi, 1) \cdot \nabla_{x, t} P\right| /\left|\nabla_{x, t} P\right| \lesssim R^{-1 / 2+\delta^{\prime}}$, the desired estimate (3.5) follows.

\subsection{Linear refined Strichartz estimates}

Before proving Theorem 2.6, we consider the linear and bilinear refined Strichartz estimates. We first prove the linear refined Strichartz estimates (Proposition 3.6) and then prove the bilinear estimates (Proposition 3.10) by using Proposition 3.6.

Proposition 3.6. Let $\phi \in \mathcal{P}\left(\epsilon_{0}\right)$. Suppose that $f$ is concentrated on the wave packets from $T_{Z}\left(R^{-1 / 2+\delta^{\prime}}\right)$ and $\widehat{f}$ is supported on $B^{2}(0,1)$. Let $Q_{1}, Q_{2}, \ldots$ be lattice cubes of side length $R^{1 / 2}$ in $B^{3}(0, R)$. Suppose that $M$ cubes $Q_{j}$ are contained in $B^{2}(0, R) \times\left[t_{0}, t_{0}+R^{1 / 2}\right]$ for each $t_{0} \in R^{1 / 2} \mathbb{Z} \cap[0, R]$, and for each $Q_{j}$,

$$
\left\|e^{i t \phi} f\right\|_{L^{6}\left(Q_{j}\right)} \text { is essentially constant. }
$$

Then, for any $\epsilon>0$, there exist constants $C_{\epsilon}, C \geq 1$ such that

$$
\left\|e^{i t \phi} f\right\|_{L^{6}\left(\bigcup_{j} Q_{j}\right)} \leq C_{\epsilon} R^{-1 / 6+\epsilon+C \delta^{\prime}} M^{-1 / 3}\|f\|_{2} .
$$

We start by recalling the $l^{2}$-decoupling inequality for an elliptic paraboloid, which was obtained by Bourgain and Demeter 4 . 
Theorem 3.7. Suppose that $\widehat{g}$ is supported in a $\sigma$-neighborhood of an elliptic paraboloid $S$ in $\mathbb{R}^{2}$. Let $\tau$ be rectangles of dimensions $\sigma^{1 / 2} \times \sigma$, which cover a $\sigma$-neighborhood of $S$. If $\widehat{g}_{\tau}=\widehat{g} \chi_{\tau}$, then for $\epsilon>0$ and $2 \leq p \leq 6$, we have

$$
\|g\|_{L^{p}\left(\mathbb{R}^{2}\right)} \leq C_{\epsilon} \sigma^{-\epsilon}\left(\sum_{\tau}\left\|g_{\tau}\right\|_{L^{p}\left(\mathbb{R}^{2}\right)}^{2}\right)^{1 / 2}
$$

Let us consider the wave packet decomposition

$$
f=\sum_{T} f_{T}
$$

such that the Fourier support of $f_{T}$ is contained in a ball of radius $R^{-1 / 4}$ and $f_{T}$ is essentially supported on a ball of radius $R^{3 / 4}$. Then, $e^{i t \phi} f_{T}$ restricted to the ball $B^{3}(0, R)$ is essentially supported on a tube $T$ of dimensions $R^{3 / 4} \times R^{3 / 4} \times R$. Since $f$ is concentrated on the wave packets from $T_{Z}\left(R^{-1 / 2+\delta^{\prime}}\right)$ for some $Z$, we can apply Theorem 3.7 and obtain the following.

Proposition 3.8. Let $\phi \in \mathcal{P}\left(\epsilon_{0}\right)$ for sufficiently small $\epsilon_{0}>0$. Let $f=\sum_{T} f_{T}$ be as stated above. Suppose that $f$ is concentrated on the wave packets from $T_{Z}\left(R^{-1 / 2+\delta^{\prime}}\right)$ for some $Z=Z(P)$ and suppose that $Q$ is a cube of side length $R^{1 / 2}$ contained in the $2 R^{1 / 2+\delta^{\prime}}$ neighborhood of $Z$. Then,

$$
\left\|e^{i t \phi} f\right\|_{L^{6}(Q)} \leq C_{\epsilon} R^{\epsilon}\left(\sum_{T}\left\|e^{i t \phi} f_{T}\right\|_{L^{6}\left(\omega_{Q}\right)}^{2}\right)^{1 / 2}+O\left(R^{-N}\right)\|f\|_{2} .
$$

Here, $\omega_{Q}(z)=\left(1+R^{-1 / 2}\left|z-c_{Q}\right|\right)^{-100}$, where $c_{Q}$ is the center of the cube $Q$.

Proof. Let $\psi \in \mathcal{S}\left(\mathbb{R}^{3}\right)$ such that $\psi=1$ on $B^{3}(0,1)$ and $\widehat{\psi}$ is supported on $B^{3}(0,1)$. By letting $\psi_{Q}(z)=\psi\left(C R^{-1 / 2}\left(z-c_{Q}\right)\right)$ for some constant $C>0$ such that $\psi=1$ on $Q$, we get

$$
\left\|e^{i t \phi} f\right\|_{L^{6}(Q)} \leq\left\|\psi_{Q} e^{i t \phi} f\right\|_{L^{6}\left(\mathbb{R}^{3}\right)} .
$$

Since $f$ is concentrated on the wave packets from $T_{Z}\left(R^{-1 / 2+\delta^{\prime}}\right)$, it suffices to consider the wave packets that are contained in the $R^{1 / 2+\delta^{\prime}}$-neighborhood of a plane $W=T_{z} Z(P)$. We claim that

$$
\left\|\psi_{Q} e^{i t \phi} f\right\|_{L^{6}(W)} \leq C_{\epsilon} R^{\epsilon / 2}\left(\sum_{T}\left\|\psi_{Q} e^{i t \phi} f_{T}\right\|_{L^{6}(W)}^{2}\right)^{1 / 2}+O\left(R^{-N}\right)\|f\|_{2} .
$$

By assuming (3.11), we prove (3.10). By integrating along the $W^{\perp}$ axis and using Minkowski's inequality and Fubini's theorem, we obtain

$$
\left\|e^{i t \phi} f\right\|_{L^{6}(Q)} \leq C_{\epsilon} R^{\epsilon / 2}\left(\sum_{T}\left\|\psi_{Q} e^{i t \phi} f_{T}\right\|_{L^{6}\left(\mathbb{R}^{3}\right)}^{2}\right)^{1 / 2}+O\left(R^{-N}\right)\|f\|_{2} .
$$


Here, we use the fact that the number of tubes $T$ intersecting $Q$ is $\lesssim R^{\epsilon / 100}$. Since $\psi_{Q}$ decays rapidly outside $Q$, we get the desired result (3.10).

Now, we prove (3.11). It suffices to show that the restriction of $\mathcal{F}\left(\psi_{Q} e^{i t \phi} f\right)$ to $W$ is contained in an $R^{-1 / 4}$-neighborhood of an elliptic paraboloid. Since $\mathcal{F}\left(\psi_{Q}\right)$ is supported on $B^{3}\left(0, R^{-1 / 2}\right)$, it suffices to consider the restriction of $\mathcal{F}\left(e^{i t \phi} f\right)$ to $W$. Let $\mathbf{n}$ be the unit normal vector of $W$. Since $\widehat{f}$ is supported on $B^{2}(0,1)$, we have $\left|\mathbf{n} \cdot e_{3}\right|<1 / 2$. By rotation and dilation, we may assume that $\mathbf{n}=\mathbf{n}^{\prime} /\left|\mathbf{n}^{\prime}\right|$, where $\mathbf{n}^{\prime}=\left(0,1, n_{3}\right)$ for some $\left|n_{3}\right| \lesssim 1$. Since $\partial_{\xi_{2}}^{2} \phi \neq 0$ on $B^{2}(0,1)$, by the implicit function theorem, there is a function $g \in \mathcal{C}_{0}^{1}((-1,1))$ such that

$$
\left(-\nabla \phi\left(\xi_{1}, g\left(\xi_{1}\right)\right), 1\right) \cdot \mathbf{n}=0
$$

and equivalently, $n_{3}=\partial_{\xi_{2}} \phi\left(\xi_{1}, g\left(\xi_{1}\right)\right)$.

Note that $|(-\nabla \phi(\xi), 1) \cdot \mathbf{n}|=\left|-\partial_{\xi_{2}} \phi+n_{3}\right| \lesssim R^{-1 / 4}$ on the support of supp $\widehat{f}$. Since $\partial_{\xi_{2}}^{2} \phi \neq 0$, by the mean value theorem, we have

$$
\left|\xi_{2}-g\left(\xi_{1}\right)\right| \lesssim R^{-1 / 4}
$$

Therefore, we may write

$$
(\xi, \phi(\xi))=\left(\xi_{1}, 0, \widetilde{\phi}\left(\xi_{1}\right)\right)+\xi_{2} \mathbf{n}^{\prime}+\mathcal{E}(\xi) e_{3},
$$

where $\widetilde{\phi}\left(\xi_{1}\right)=\phi\left(\xi_{1}, g\left(\xi_{1}\right)\right)-n_{3} g\left(\xi_{1}\right)$ and $\mathcal{E}(\xi)=\phi(\xi)-\phi\left(\xi_{1}, g\left(\xi_{1}\right)\right)-n_{3}\left(\xi_{2}-g\left(\xi_{1}\right)\right)$. By (3.13) and the mean value theorem, it is easy to see that $\mathcal{E}(\xi)=O\left(R^{-1 / 2}\right)$. Moreover, by (3.12), $\partial_{\xi_{1}} \widetilde{\phi}\left(\xi_{1}\right)=\left(\partial_{\xi_{1}} \phi\right)\left(\xi_{1}, g\left(\xi_{1}\right)\right)$; hence, $\partial_{\xi_{1}}^{2} \widetilde{\phi}\left(\xi_{1}\right)=\left(\partial_{\xi_{1}}^{2} \phi\right)\left(\xi_{1}, g\left(\xi_{1}\right)\right)+O\left(\epsilon_{0}\right)$ whenever $\phi \in \mathcal{P}\left(\epsilon_{0}\right)$ for sufficiently small $\epsilon_{0}>0$. Thus, the curve parameterized by $\xi_{1} \rightarrow \widetilde{\phi}\left(\xi_{1}\right)$ is contained in an $R^{-1 / 2}$-neighborhood of the elliptic paraboloid. By Theorem 3.7, we obtain the desired bound (3.11).

Using Proposition 3.8, we can prove Proposition 3.6 by induction.

Proof of Proposition 3.6. Let us set $\mathcal{A}_{0}:=\mathcal{A}_{\xi_{0}}^{R^{-1 / 4}}$ for simplicity. Recalling (3.9), for each tube $T$, we choose a cube $Q_{T}$ of side length $R^{1 / 2}$ that is contained in $\mathcal{A}_{0}(T)$. We decompose $Q_{T}$ into horizontal strips $S^{\prime}$ of height $R^{1 / 4}$ in $Q_{T}$ such that $Q_{T}=\cup S^{\prime}$. Furthermore, each strip $S^{\prime}$ is decomposed into cubes $Q^{\prime}$ of side length $R^{1 / 4}$. We set $T^{\prime}:=\mathcal{A}_{0}^{-1}\left(Q^{\prime}\right)$, which is a tube of size $\sim R^{1 / 2} \times R^{1 / 2} \times R^{3 / 4}$ such that the union of all $T^{\prime}$ covers $T$ (see Figure 3.1.

By dyadic pigeonholing on the size of $\left\|f_{T}\right\|_{2}$, we may assume that $\left\|f_{T}\right\|_{2}$ is essentially constant for each tube $T$. In fact, the number of $h$ is only $C \log R$ such that $\left\|f_{T}\right\|_{2} \sim h$ since it is negligible if $\left\|f_{T}\right\|_{2} \leq R^{-N}\|f\|_{2}$. Therefore, we choose one of $h$ such that $\left\|f_{T}\right\|_{2}$ 


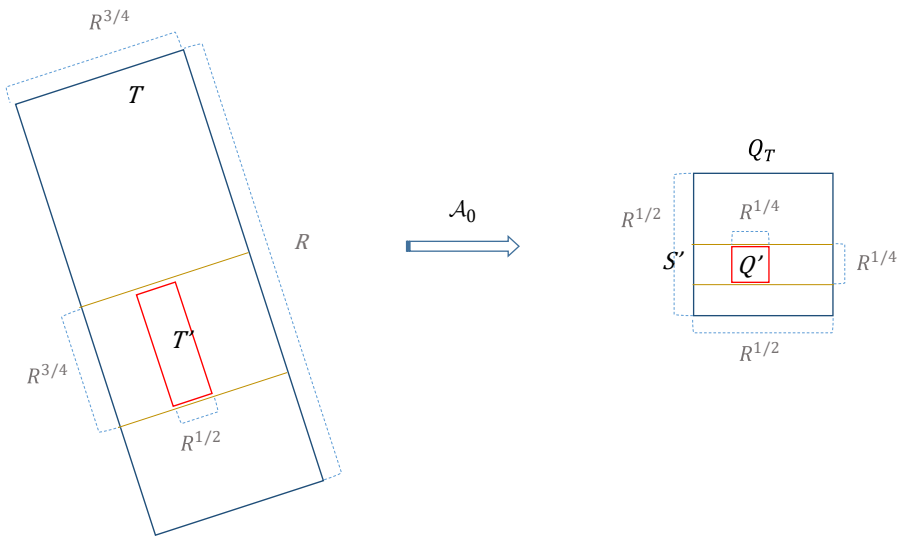

Figure 3.1

is essentially constant for a fraction $\sim 1 /(\log R)$ of tubes $T$. For a fixed such tube $T$, we again perform dyadic pigeonholing on the size of $\left\|e^{i t \phi} f_{T}\right\|_{L^{6}\left(T^{\prime}\right)}$ such that

$$
\left\|e^{i t \phi} f_{T}\right\|_{L^{6}\left(T^{\prime}\right)} \text { is essentially constant, }
$$

which is greater than $R^{-N}\|f\|_{2}$ since this part can be absorbed in the error term in (3.10) if the constant is less than $R^{-N}\|f\|_{2}$. We sort $T^{\prime}$ further according to the number of $T^{\prime}$ arranged along the short axis of $T$. Precisely, we may assume that

$$
\#\left\{T^{\prime}: T^{\prime} \subset \mathcal{A}_{0}^{-1}\left(S^{\prime}\right)\right\} \sim M^{\prime}
$$

for a dyadic number $M^{\prime}$. For simplicity, by abuse of notation, we denote such tubes by $T^{\prime}$. Then, it follows that

$$
\left\|e^{i t \phi} f\right\|_{L^{6}\left(Q_{j}\right)} \lesssim(\log R)^{3}\left\|\sum_{T} \sum_{T^{\prime}} \chi_{\cup T^{\prime}} e^{i t \phi} f_{T}\right\|_{L^{6}\left(Q_{j}\right)}
$$

for a fraction $\sim 1 /(\log R)^{3}$ of all cubes $Q_{j}$ in $\bigcup_{j} Q_{j}$. Finally, we sort the cubes $Q_{j}$ further such that $Q_{j}$ satisfies (3.15) and each $Q_{j}$ is contained in $\sim \mu$ tubes $T$ such that $Q_{j} \subset T^{\prime} \subset T$. Let $\mathcal{Q}$ denote the set of such cubes $Q_{j}$; then, we see that $\# \mathcal{Q} \gtrsim(\log R)^{-4} M$ by dyadic pigeonholing. By (3.7), we have $\left\|e^{i t \phi} f\right\|_{L^{6}\left(\cup_{j} Q_{j}\right)}^{6} \lesssim(\log R)^{4}\left\|e^{i t \phi} f\right\|_{L^{6}\left(\cup_{Q_{j} \in \mathcal{Q}} Q_{j}\right)}$. Hence, it suffices to consider cubes $Q_{j} \in \mathcal{Q}$.

By 3.15, 3.10, and Hölder's inequality, we get

$$
\left\|e^{i t \phi} f\right\|_{L^{6}\left(Q_{j}\right)} \leq C_{\varepsilon}(\log R)^{4} R^{\varepsilon} \mu^{1 / 3}\left(\sum_{T}\left\|\chi \cup T^{\prime} e^{i t \phi} f_{T}\right\|_{L^{6}\left(\omega_{Q_{j}}\right)}^{6}\right)^{1 / 6}+O\left(R^{-N}\right)\|f\|_{2} .
$$

Since $T^{\prime}=\mathcal{A}_{0}^{-1}\left(Q^{\prime}\right)$ for some cube $Q^{\prime}$ of side length $R^{1 / 4}$, by $(3.3)$, we have

$$
\sum_{Q_{j}}\left\|\chi_{\cup T^{\prime}} e^{i t \phi} f_{T}\right\|_{L^{6}\left(\omega_{Q_{j}}\right)}^{6} \lesssim R^{-1 / 2}\left\|e^{i t \widetilde{\phi}} \widetilde{f}_{T}\right\|_{L^{6}\left(\cup Q^{\prime}\right)}^{6}
$$


where $\mathcal{F}\left(\widetilde{f}_{T}\right)$ is given by 3.4 for $f_{T}$ in place of $f$. Then, $\mathcal{F}\left(\widetilde{f}_{T}\right)$ is supported on $B^{2}(0,1)$ and $\left\|\tilde{f}_{T}\right\|_{2}=\left\|f_{T}\right\|_{2}$. Combining this with (3.16), we have

$$
\left\|e^{i t \phi} f\right\|_{L^{6}\left(\bigcup_{j} Q_{j}\right)} \leq C_{\varepsilon} R^{-1 / 12+2 \varepsilon} \mu^{1 / 3}\left(\sum_{T}\left\|e^{i t \widetilde{\phi}} \widetilde{f}_{T}\right\|_{L^{6}\left(\cup Q^{\prime}\right)}^{6}\right)^{1 / 6}+O\left(R^{-N}\right)\|f\|_{2} .
$$

By the choice of (3.14) combined with 3.3 for $f=f_{T}$, we observe that $\left\|e^{i t \widetilde{\phi}} \widetilde{f}_{T}\right\|_{L^{6}\left(Q^{\prime}\right)}$ is essentially constant for each $Q^{\prime}$. Since $\phi \in \mathcal{P}\left(\epsilon_{0}\right)$, we have $\widetilde{\phi} \in \mathcal{P}\left(\epsilon_{0}\right)$ by Lemma 3.2 provided that $R^{-1 / 2} \leq \rho_{1}$. Thus, by applying the induction hypothesis to the right-hand side of (3.17) with $R^{1 / 2}$ and $M^{\prime}$ instead of $R$ and $M$, respectively, we have

$$
\left\|e^{i t \phi} f\right\|_{L^{6}\left(\bigcup_{j} Q_{j}\right)} \leq C_{\varepsilon} R^{-\frac{1}{6}+2 \epsilon+\frac{1}{2} C \delta^{\prime}} \mu^{1 / 3}\left(M^{\prime}\right)^{-1 / 3}\left(\sum_{T}\left\|f_{T}\right\|_{2}^{6}\right)^{1 / 6}
$$

Since $\left\|f_{T}\right\|_{2}$ is essentially constant, it is easy to see that $\left(\sum_{T}\left\|f_{T}\right\|_{2}^{6}\right)^{1 / 6} \lesssim(\# T)^{-1 / 3}\|f\|_{2}$. We claim that

$$
M \mu \lesssim(\log R)^{4} M^{\prime} \# T
$$

Once we have 3.19, 3.18 is, in turn, bounded by

$$
\left\|e^{i t \phi} f\right\|_{L^{6}\left(\bigcup_{j} Q_{j}\right)} \leq C_{\varepsilon} R^{-\frac{1}{6}+3 \varepsilon+\frac{1}{2} C \delta^{\prime}} M^{-1 / 3}\|f\|_{2} .
$$

Taking $\varepsilon=\epsilon / C$ for sufficiently large $C>0$ gives the desired bound (3.8).

Now, we prove 3.19 . Let $S^{\left(t_{0}\right)}$ be the strip given by

$$
S^{\left(t_{0}\right)}=\mathbb{R}^{2} \times\left[t_{0}, t_{0}+R^{1 / 2}\right]
$$

for some $t_{0} \in[0, R] \cap R^{1 / 2} \mathbb{Z}$. It suffices to consider cubes $Q_{j}$ arranged along the strip $S^{\left(t_{0}\right)}$. By the choice of $Q_{j}, T$, and $T^{\prime}$, we note that

$$
(\log R)^{-4} M \mu \lesssim \#\left\{\left(Q_{j}, T\right): Q_{j} \subset T^{\prime} \cap S^{\left(t_{0}\right)} \text { for some } T^{\prime} \subset T\right\}
$$

Note that the angle between the long axis of $T^{\prime}$ and the vectors contained in the $x$-plane $\mathbb{R}^{2}$ is away from 0 . Hence, each tube $T^{\prime}$ contains at most a finite number of cubes $Q_{j}$ in $S^{\left(t_{0}\right)}$. Similarly, since $T^{\prime} \subset \mathcal{A}_{0}^{-1}\left(S^{\prime}\right)$ for some $S^{\prime}$, the number of $S^{\prime}$ such that $S^{\left(t_{0}\right)} \cap \mathcal{A}_{0}^{-1}\left(S^{\prime}\right) \neq \emptyset$ is at most $C$. Hence, the number of $Q_{j}$ is smaller than $C$ times that of $T^{\prime}$ such that $T^{\prime} \subset \mathcal{A}_{0}^{-1}\left(S^{\prime}\right)$. Therefore, $\# Q_{j} \leq C M^{\prime}$. Thus, the right-hand side of 3.20 is bounded by $M^{\prime} \# T$, which gives the desired bound 3.19 . 


\subsection{Bilinear refined Strichartz estimates}

Now, we establish the bilinear refined Strichartz estimates using Proposition 3.6 .

Proposition 3.9. Let $\phi \in \mathcal{P}\left(\epsilon_{0}\right), R^{-1 / 2} \leq r \leq 1$, and $K$ be a sufficiently large constant. Suppose that the Fourier supports of $f, g$ are contained in $B^{2}\left(\xi_{0}, r\right)$ and separated by $K^{-1} r$, and $f, g$ are concentrated on the wave packets from $T_{Z}\left(R^{-1 / 2+\delta^{\prime}}\right)$ for $Z=Z(P)$ with $\delta^{\prime} \leq 100 \delta$. Let $Q_{1}, \ldots, Q_{M}$ be cubes of side length $R^{1 / 2}$ contained in $B_{R}$ and

$$
\left\|\left|e^{i t \phi} f e^{i t \phi} g\right|^{1 / 2}\right\|_{L^{6}\left(Q_{j}\right)} \text { is essentially constant }
$$

for each $Q_{j}$. Then, for any $\epsilon>0$, there exist $C_{\epsilon}, C \geq 1$ such that

$$
\left\|\left|e^{i t \phi} f e^{i t \phi} g\right|^{1 / 2}\right\|_{L^{6}\left(\bigcup_{j=1}^{M} Q_{j}\right)} \leq C_{\epsilon} r^{-1 / 6} M^{-1 / 6} R^{-1 / 6+\epsilon+C \delta^{\prime}}\|f\|_{2}^{1 / 2}\|g\|_{2}^{1 / 2} .
$$

Before proving Proposition 3.9, we first consider the special case $r=1$ of Proposition 3.9 as follows, which is a direct consequence of Proposition 3.6 .

Proposition 3.10. Let $\phi \in \mathcal{P}\left(\epsilon_{0}\right)$. Suppose that the Fourier supports of $f, g$ are contained in $B^{2}(0,1)$ and separated by $K^{-1}$, and $f$ and $g$ are concentrated on the wave packets from $T_{Z}\left(R^{-1 / 2+\delta^{\prime}}\right)$ for $\delta^{\prime} \leq 100 \delta$. Suppose that $Q_{1}, \ldots, Q_{M}$ are cubes of side length $R^{1 / 2}$, and $\left\|e^{i t \phi} f\right\|_{L^{6}\left(Q_{j}\right)}$ and $\left\|e^{i t \phi} g\right\|_{L^{6}\left(Q_{j}\right)}$ are essentially constant for each $Q_{j}$. Then, for any $\epsilon>0$, there exist $C_{\epsilon}, C \geq 1$ such that

$$
\left\|\left|e^{i t \phi} f e^{i t \phi} g\right|^{1 / 2}\right\|_{L^{6}\left(\bigcup_{j} Q_{j}\right)} \leq C_{\epsilon} R^{-1 / 6+\epsilon+C \delta^{\prime}} M^{-1 / 6}\|f\|_{2}^{1 / 2}\|g\|_{2}^{1 / 2}
$$

Proof. Let us consider the wave packet decompositions $f=\sum_{T} f_{T}$ and $g=\sum_{\widetilde{T}} g_{\widetilde{T}}$ (see (3.9) ). By repeating the pigeonholing arguments as in the proof of Proposition 3.6, we can choose $T_{f}, T_{f}^{\prime}, M_{f}^{\prime}, \mu_{f}$ and $T_{g}, T_{g}^{\prime}, M_{g}^{\prime}, \mu_{g}$, respectively, in place of $T, T^{\prime}, M, \mu$. Then, by pigeonholing, there are $C(\log R)^{-8} M$ cubes $Q_{j}$ such that 3.18 holds for $f$ and $g$ simultaneously. We claim that

$$
M \mu_{f} \mu_{g} \lesssim M_{f}^{\prime} M_{g}^{\prime} \# T_{f} \# T_{g}
$$

First, by assuming (3.24), we prove 3.23). By Hölder's inequality,

$$
\left\|\left|e^{i t \phi} f e^{i t \phi} g\right|^{1 / 2}\right\|_{L^{6}\left(\bigcup_{j} Q_{j}\right)} \leq\left\|e^{i t \phi} f\right\|_{L^{6}\left(\bigcup_{j} Q_{j}\right)}^{1 / 2}\left\|e^{i t \phi} g\right\|_{L^{6}\left(\bigcup_{j} Q_{j}\right)}^{1 / 2} .
$$

We apply $(3.18)$ to the right-hand side and get

$$
\left\|\left|e^{i t \phi} f e^{i t \phi} g\right|^{1 / 2}\right\|_{L^{6}\left(\bigcup_{j} Q_{j}\right)} \leq C_{\epsilon} R^{-\frac{1}{6}+\epsilon+\frac{1}{2} C \delta^{\prime}}\left(\mu_{f} \mu_{g}\right)^{1 / 6}\left(\# T_{f} \# T_{g} M_{f}^{\prime} M_{g}^{\prime}\right)^{-1 / 6}\|f\|_{2}^{1 / 2}\|g\|_{2}^{1 / 2} .
$$

Substituting (3.24), we obtain the desired bound (3.23). 
Now, we prove (3.24). For each fixed $T_{f}$ and $T_{g}$, we first consider the intersection of $T_{f}^{\prime} \subset T_{f}$ and $T_{g}^{\prime} \subset T_{g}$. Since the angle between $T_{f}^{\prime}$ and $T_{g}^{\prime}$ is about $1 / K$, the intersection of $T_{f}^{\prime}$ and $T_{g}^{\prime}$ is essentially a cube of side length $C_{K} R^{1 / 2}$. Therefore, if cubes $Q_{j}$ are contained in $\left(\cup T_{f}^{\prime}\right) \cap\left(\cup T_{g}^{\prime}\right)$, then the number of such $Q_{j}$ is at most $C M_{f}^{\prime} M_{g}^{\prime}$.

Recall that $\mu_{f}$ is the number of $T_{f}$ containing $Q_{j}$ such that $Q_{j} \subset T_{f}^{\prime}$ and $\mu_{g}$ similarly. Considering all pairs $\left\{T_{f}, T_{g}, Q_{j}\right\}$ such that $Q_{j} \subset T_{f}^{\prime} \cap T_{g}^{\prime}$ for some $T_{f}^{\prime} \subset T_{f}$ and $T_{g}^{\prime} \subset T_{g}$, we obtain (3.24).

Now, we prove Proposition 3.9. If $r \sim 1$, Proposition 3.9 is a direct consequence of Proposition 3.10. Hence, we are only concerned with $R^{-1 / 2} \leq r \ll 1$. In this case, Proposition 3.9 follows from Proposition 3.10 via rescaling in Lemma 3.3.

Proof of Proposition 3.9. Let $\phi \in \mathcal{P}\left(\epsilon_{0}\right)$. By $3.2, \mathcal{A}_{\xi_{0}}^{r}\left(B_{R}\right)$ is a tube of dimensions $C r R \times$ $C r R \times r^{2} R$ for a constant $C \geq 1$. Let us set

$$
P_{j}=\mathcal{A}_{\xi_{0}}^{r}\left(Q_{j}\right)
$$

Since $Q_{j}$ is a cube of side length $R^{1 / 2}, P_{j}$ is a tube of dimensions $C r R^{1 / 2} \times C r R^{1 / 2} \times r^{2} R^{1 / 2}$ contained in a larger tube $\mathcal{A}_{\xi_{0}}^{r}\left(B_{R}\right)$.

We define $\tilde{f}$ and $\widetilde{g}$ by 3.4 for $f$ and $g$, respectively, with $r=\rho$. Further, let $\widetilde{\phi}=\phi_{\xi_{0}}^{r}$. By Lemma 3.2 , we see that $\widetilde{\phi} \in \mathcal{P}\left(\epsilon_{0}\right)$ by taking sufficiently small $r \leq \rho_{1}$. Moreover, by Lemma 3.3 , we have

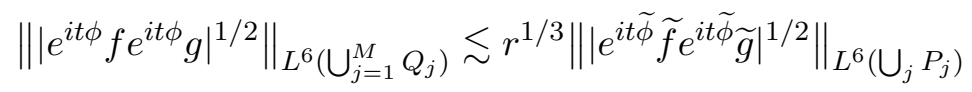

such that $\|\widetilde{f}\|_{2}=\|f\|_{2},\|\widetilde{g}\|_{2}=\|g\|_{2}$ and the Fourier supports of $\widetilde{f}$ and $\widetilde{g}$ are contained in $B^{2}(0,1)$ and separated by $K^{-1}$. Moreover, by Lemma 3.5 . $\widetilde{f}$ and $\widetilde{g}$ are $r^{-1} R^{-1 / 2+\delta^{\prime}}$. concentrated in the wave packets from $T_{Z}\left(r^{-1} R^{-1 / 2+\delta^{\prime}}\right)$ associated with $\widetilde{\phi}$ for $Z=Z(\widetilde{P})$ for some polynomial $\widetilde{P}=P \circ\left(\mathcal{A}_{\xi_{0}}^{r}\right)^{-1}$. Thus, to prove 3.22 , it suffices to show that

$$
\left\|\left|e^{i t \widetilde{\phi}} \widetilde{f} e^{i t \widetilde{\phi}} \widetilde{g}\right|^{1 / 2}\right\|_{L^{6}\left(\bigcup_{j} P_{j}\right)} \leq C_{\epsilon} r^{-1 / 2} M^{-1 / 6} R^{-1 / 6+\epsilon+C \delta^{\prime}}\|\widetilde{f}\|_{2}^{1 / 2}\|\widetilde{g}\|_{2}^{1 / 2}
$$

Now, we make couple of reductions by pigeonholing. For this purpose, let us set

$$
r_{1}=r^{2} R \quad \text { and } \quad r_{2}=r R^{1 / 2} .
$$

Recall that $\mathcal{A}_{\xi_{0}}^{r}\left(B_{R}\right)$ is a tube of dimensions $C r^{-1} r_{1} \times C r^{-1} r_{1} \times r_{1}$. Now, we decompose $\mathcal{A}_{\xi_{0}}^{r}\left(B_{R}\right)$ into cubes $Q_{r_{1}}$ of side length $r_{1}$. For each fixed cube $Q_{r_{1}}$, we consider cubes $Q_{r_{2}}^{\prime}$ of side length $r_{2}$ contained in $Q_{r_{1}}$ such that $\left\{Q_{r_{2}}^{\prime}\right\}$ covers $\left(\bigcup_{j} P_{j}\right) \cap Q_{r_{1}}$. By dyadic pigeonholing on the size of $\left\|e^{i t \widetilde{\phi}} \widetilde{f}\right\|_{L^{2}\left(Q_{r_{2}}^{\prime}\right)}$ and $\left\|e^{i t \widetilde{\phi}} \widetilde{g}\right\|_{L^{2}\left(Q_{r_{2}}^{\prime}\right)}$, we may sort $Q_{r_{2}}^{\prime}$, which satisfy

$$
\left\|e^{i t \widetilde{\phi}} \widetilde{f}\right\|_{L^{2}\left(Q_{r_{2}}^{\prime}\right)}, \quad\left\|e^{i t \widetilde{\phi}} \widetilde{g}\right\|_{L^{2}\left(Q_{r_{2}}^{\prime}\right)} \text { are essentially constant. }
$$


Let $\mathcal{Q}_{r_{2}}^{\prime}$ be the collection of such cubes $Q_{r_{2}}^{\prime}$. We also choose a subcollection of $\left\{Q_{r_{1}}\right\}$ that satisfy

$$
\left\|\left|e^{i t \tilde{\phi}} \widetilde{f} e^{i t \widetilde{\phi}} \widetilde{g}\right|^{1 / 2}\right\|_{L^{6}\left(\bigcup_{Q_{r_{2}}^{\prime} \in \mathcal{Q}_{r_{2}}^{\prime}} Q_{r_{2}}^{\prime}\right)} \text { is essentially constant }
$$

for $Q_{r_{2}}^{\prime} \in \mathcal{Q}_{r_{2}}^{\prime}$ such that $Q_{r_{2}}^{\prime} \subset Q_{r_{1}}$, and also satisfy

$$
\left\|e^{i t \widetilde{\phi} \widetilde{f}}\right\|_{L^{2}\left(R^{2 a} Q_{r_{1}}\right)}, \quad\left\|e^{i t \widetilde{\phi}} \widetilde{g}\right\|_{L^{2}\left(R^{2 a} Q_{r_{1}}\right)} \text { are essentially constant. }
$$

Here, $R^{2 a} Q_{r_{1}}$ is a cube that has the same center as $Q_{r_{1}}$ and the side length is $R^{2 a} r_{1}$ for a sufficiently small constant $a=a(\epsilon)$, which will be determined later. We denote such a collection of $Q_{r_{1}}$ by $\mathcal{Q}_{r_{1}}$. We also denote by $\mathcal{P}=\mathcal{P}\left(r_{1}, r_{2}\right)$ the collection of tubes $P_{j}$ that intersect with $Q_{r_{2}}^{\prime} \subset Q_{r_{1}}$ for $Q_{r_{2}}^{\prime} \in \mathcal{Q}_{r_{2}}^{\prime}$ and $Q_{r_{1}} \in \mathcal{Q}_{r_{1}}$. By dyadic pigeonholing, we have

$$
\# \mathcal{P} \gtrsim(\log R)^{-5} M \text {. }
$$

We put

$$
M_{1}=\#\left\{Q_{r_{1}} \in \mathcal{Q}_{r_{1}}\right\}, \quad M_{2}=\#\left\{Q_{r_{2}}^{\prime} \in \mathcal{Q}_{r_{2}}^{\prime}\right\} .
$$

Since the number of $P_{j} \in \mathcal{P}$ contained in a cube $Q_{r_{2}}^{\prime}$ is at most $r^{-1}$, it is easy to see that

$$
(\log R)^{-5} M \lesssim r^{-1} M_{1} M_{2} .
$$

Thus, from (3.21) and 3.30 , we get

$$
\left\|\left|e^{i t \widetilde{\phi}} \widetilde{f} e^{i t \widetilde{\phi}} \widetilde{g}\right|^{1 / 2}\right\|_{L^{6}\left(\bigcup_{j} P_{j}\right)}^{6} \lesssim(\log R)^{5}\left\|\left|e^{i t \widetilde{\phi}} \widetilde{f} e^{i t \widetilde{\phi}} \widetilde{g}\right|^{1 / 2}\right\|_{L^{6}\left(\bigcup_{P_{j} \in \mathcal{P}} P_{j}\right)}^{6} .
$$

By 3.28$)$, for any $Q_{r_{1}} \in \mathcal{Q}_{r_{1}}$ and $\cup Q_{r_{2}}^{\prime} \subset Q_{r_{1}}$, we have

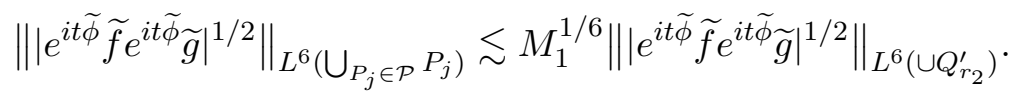

The right-hand side of 3.33 can be estimated by applying the induction hypothesis. By Lemma 2.2, we have $\widetilde{f}=\sum_{T} \widetilde{f}_{T}$ and $\widetilde{g}=\sum_{T^{\prime}} \widetilde{g}_{T^{\prime}}$. Here, the Fourier transforms of $\widetilde{f}_{T}$ and $\widetilde{g}_{T^{\prime}}$ are supported on balls of radius $r_{1}^{-1 / 2}$, and $e^{i t \widetilde{\phi}} \widetilde{f}_{T}$ and $e^{i t \tilde{\phi}} \widetilde{g}_{T}$ restricted to $B^{3}\left(0, r_{1}\right)$ are essentially supported on tubes $T, T^{\prime}$ of dimensions $\sim r_{1}^{1 / 2} \times r_{1}^{1 / 2} \times r_{1}$.

Note that it suffices to consider the wave packets that intersect with $Q_{r_{1}}$. Indeed, let us fix $t_{0}$ such that $\left(x_{0}, t_{0}\right) \in Q_{r_{1}}$ for some $x_{0}$ and denote the slice of $Q_{r_{1}}$ by

$$
Q_{r_{1}}^{\left(t_{0}\right)}=Q_{r_{1}} \cap\left\{t=t_{0}\right\}
$$

Since $\cup Q_{r_{2}}^{\prime} \subset Q_{r_{1}}$, to estimate the right-hand side of $(3.33)$, it suffices to consider the wave packets $T, T^{\prime}$ intersecting with $R^{a} Q_{r_{1}}^{\left(t_{0}\right)}$, which is an $R^{a}$-neighborhood of $Q_{r_{1}}^{\left(t_{0}\right)}$. Therefore,

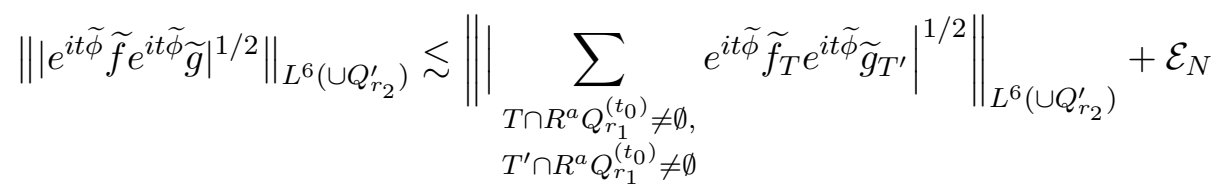


where $\mathcal{E}_{N}=O\left(R^{-N}\right)\|f\|_{2}^{1 / 2}\|g\|_{2}^{1 / 2}$ for sufficiently large $N$. Under the assumption (3.27), we apply $(3.23)$ for $R=r_{1}$ to the above inequality and using Plancherel's theorem, we obtain

$$
\left\|\left|e^{i t \widetilde{\phi}} \widetilde{f} e^{i t \widetilde{\phi}} \widetilde{g}\right|^{1 / 2}\right\|_{L^{6}\left(\cup Q_{r_{2}}^{\prime}\right)} \leq C_{\varepsilon} r_{1}^{-1 / 6+\varepsilon+C \delta^{\prime}} M_{2}^{-1 / 6}\left\|\sum_{T} e^{i t_{0} \widetilde{\phi}} \widetilde{f}_{T}\right\|_{L_{x}^{2}}^{1 / 2}\left\|\sum_{T^{\prime}} e^{i t_{0} \widetilde{\phi}} \widetilde{g}_{T^{\prime}}\right\|_{L_{x}^{2}}^{1 / 2}+\mathcal{E}_{N}
$$

where the summation is taken over $T$ and $T^{\prime}$ satisfying $T \cap R^{a} Q_{r_{1}}^{\left(t_{0}\right)} \neq \emptyset$ and $T^{\prime} \cap R^{a} Q_{r_{1}}^{\left(t_{0}\right)} \neq$ $\emptyset$.

Note that the length $C r_{1}^{1 / 2}$ of the short axis of a tube $T$ is smaller than the side length $R^{a} r_{1}$ of the cube $R^{a} Q_{r_{1}}$. Hence, the intersection of the tube $T$ and $Q_{r_{1}}^{\left(t_{0}\right)}$ is contained in $R^{2 a} Q_{r_{1}} \cap\left\{t=t_{0}\right\}$ for sufficiently large $R>0$. Thus, we have

$$
\begin{aligned}
& \left\|\mid e^{i t \widetilde{\phi}} \widetilde{f} e^{\left.i t \widetilde{\phi} \widetilde{g}\right|^{1 / 2}}\right\|_{L^{6}\left(\cup Q_{r_{2}}^{\prime}\right)} \\
\leq & C_{\varepsilon} r_{1}^{-1 / 6+\varepsilon+C \delta^{\prime}} M_{2}^{-1 / 6}\left\|e^{i t_{0} \widetilde{\phi}} \widetilde{f}\right\|_{L_{x}^{2}\left(R^{2 a} Q_{r_{1}}^{\left(t_{0}\right)}\right)}^{1 / 2} \| e^{i t_{0} \widetilde{\phi} \widetilde{g} \|_{L_{x}^{2}\left(R^{2 a} Q_{r_{1}}^{\left(t_{0}\right)}\right)}^{1 / 2}+\mathcal{E}_{N} .}
\end{aligned}
$$

By taking the average with respect to $t$, we have

$$
\left\|e^{i t_{0} \widetilde{\phi} \widetilde{f}}\right\|_{L_{x}^{2}\left(R^{2 a} Q_{r_{1}}^{\left(t_{0}\right)}\right)} \leq\left(r^{2} R^{1+2 a}\right)^{-1 / 2}\left\|e^{i t \widetilde{\phi} \widetilde{f}}\right\|_{L^{2}\left(R^{2 a} Q_{r_{1}}\right)} .
$$

Since $\cup R^{2 a} Q_{r_{1}} \subset \mathcal{A}_{\xi_{0}}^{r}\left(B_{R^{1+3 a}}\right)$, by 3.29 and Lemma 3.3, we get

$$
\left\|e^{i t \widetilde{\phi} \widetilde{f}}\right\|_{L^{2}\left(R^{2 a} Q_{r_{1}}\right)} \lesssim M_{1}^{-1 / 2}\left\|e^{i t \widetilde{\phi} \widetilde{f}}\right\|_{L^{2}\left(\mathrm{~A}_{\xi_{0}}^{r}\left(B_{R^{1+3 a}}\right)\right)} \lesssim M_{1}^{-1 / 2} r\left\|e^{i t \phi} f\right\|_{L^{2}\left(B_{R^{1+3 a}}\right)} .
$$

By combining (3.35) and 3.36) with the trivial estimate $\left\|e^{i t \phi} f\right\|_{L^{2}\left(B_{R^{1+3 a}}\right)} \lesssim R^{(1+3 a) / 2}\|f\|_{2}$ via Plancherel's theorem, we have

$$
\left\|e^{i t_{0} \widetilde{\phi} \widetilde{f}}\right\|_{L_{x, t}^{2}\left(R^{2 a} Q_{r_{1}}^{\left(t_{0}\right)}\right)} \lesssim M_{1}^{-1 / 2} R^{a / 2}\|f\|_{2}
$$

Since $r_{1}=r^{2} R$ (see (3.26) $)$, disregarding the error term $\mathcal{E}_{N}, 3.34$ is bounded by

$$
\left\|\left|e^{i t \widetilde{\phi}} \widetilde{f} e^{i t \widetilde{\phi}} \widetilde{g}\right|^{1 / 2}\right\|_{L^{6}\left(\cup Q_{r_{2}}^{\prime}\right)} \leq C_{\varepsilon}\left(r^{2} R\right)^{-1 / 6+\varepsilon+C \delta^{\prime}} M_{2}^{-1 / 6} M_{1}^{-1 / 2} R^{a / 2}\|f\|_{2}^{1 / 2}\|g\|_{2}^{1 / 2} .
$$

By combining this with $(3.32)$ and $(3.33)$, and taking sufficiently small $a=a(\varepsilon)$ for $R^{-1 / 2} \leq r$, we obtain

$$
\left\|\left|e^{i t \widetilde{\phi}} \widetilde{f} e^{i t \widetilde{\phi}} \widetilde{g}\right|^{1 / 2}\right\|_{L^{6}\left(\bigcup_{j} P_{j}\right)} \leq C_{\varepsilon} R^{-1 / 6+3 \varepsilon+C \delta^{\prime}} r^{-1 / 3} M_{1}^{-1 / 3} M_{2}^{-1 / 6}\|f\|_{2}^{1 / 2}\|g\|_{2}^{1 / 2} .
$$

Therefore, using (3.31), the above is bounded by $C_{\varepsilon} R^{-1 / 6+4 \varepsilon+C \delta^{\prime}} r^{-1 / 2} M^{-1 / 6} M_{1}^{-1 / 6}\|f\|_{2}^{1 / 2}$ $\|g\|_{2}^{1 / 2}$. Since $M_{1} \geq 1$, by taking $\varepsilon \leq \epsilon / C_{1}$ for sufficiently large $C_{1} \geq 1$, we obtain (3.25). This completes the proof of 3.22 .

We conclude this section by proving Theorem 2.6 . 


\subsection{Proof of Theorem 2.6}

Let $\epsilon>0$. Assume that $\widehat{f}, \widehat{g}$ are supported on $B^{2}\left(\xi_{0}, r\right)$ for some $0<r<1$ and $\xi_{0} \in \operatorname{int} \mathbb{A}_{1}$. From (2.4), we may assume that $r \geq R^{-1 / 2}$. For a dyadic number $\lambda$, we define a set $X_{\lambda}$ by

$$
X_{\lambda}=\left\{x \in B^{2}(0, R): \sup _{0<t<R}\left|U_{\alpha} f(x) U_{\alpha} g(x)\right| \sim \lambda\right\} .
$$

It suffices to consider $R^{-N} \leq \lambda \leq R^{c}$.

We cover $N_{R^{1 / 2+\delta^{\prime}}}(Z)$ with cubes $Q_{j}, j=1, \ldots, M$, of side length $R^{1 / 2}$. Since $\mathcal{F}\left(U_{\alpha} f\right)$ is supported on a ball of radius $r$, we consider smaller cubes $Q^{\prime}=Q^{\prime}\left(Q_{j}\right)$ of side length $r^{-1} \leq R^{1 / 2}$ such that $Q^{\prime} \subset Q_{j}$. We define $S_{\lambda}$ by the union of $Q^{\prime}$ contained in $B_{R}$ such that

$$
\sup _{(x, t) \in Q^{\prime}}\left|U_{\alpha} f(x) U_{\alpha} g(x)\right| \gtrsim \lambda
$$

and all projections of $Q^{\prime}$ onto the $x$-plane $\mathbb{R}^{2}$ are boundedly overlapping. Clearly, we have

$$
\left|X_{\lambda}\right| \lesssim r\left|S_{\lambda}\right|
$$

For each $Q_{j}$, the number of $Q^{\prime}$ contained in $Q_{j}$ is at most $C\left(R^{1 / 2} / r^{-1}\right)^{2}=C R r^{2}$ since the projections of $Q^{\prime}$ onto $x$-space are finitely overlapping. Hence, by pigeonholing, we also have

$$
\left|S_{\lambda}\right| \lesssim(\log R) M R r^{-1}
$$

Now, we prove Theorem 2.6. By construction, it suffices to show that

$$
\lambda^{1 / 2}\left|X_{\lambda}\right|^{1 / 3} \leq C R^{c \delta^{\prime}}\|f\|_{2}^{1 / 2}\|g\|_{2}^{1 / 2} .
$$

After finite decomposition, we may assume that $\widehat{f}$ and $\widehat{g}$ are supported on a ball $B^{2}\left(\eta_{0}, \epsilon_{1} r\right)$ for some small $\epsilon_{1}$ and $\eta_{0} \in \operatorname{int} \mathbb{A}_{1}$ since $f$ and $g$ have Fourier supports separated by $K^{-1} r$ such that $K^{-1} \ll \epsilon_{1}$. By Lemmas 3.2 and 3.5 with $\rho=\epsilon_{1}>0$ and $\xi_{0}=\eta_{0}$, we have $f_{\epsilon_{1}}=\tilde{f}, g_{\epsilon_{1}}=\widetilde{g}$ (see (3.4) ), and $\phi_{\epsilon_{1}}=\phi_{\eta_{0}}^{\epsilon_{1}}$ for $\phi(\xi)=|\xi|^{\alpha}$ such that $\phi_{\epsilon_{1}} \in \mathcal{P}\left(\epsilon_{0}\right)$ and $f_{\epsilon_{1}}$ and $g_{\epsilon_{1}}$ have Fourier supports on a ball $B^{2}(0, r)$ separated by $\epsilon_{1}^{-1} K^{-1} r$ and concentrated on the wave packets from $T_{Z}\left(\epsilon_{1}^{-1} R^{-1 / 2+\delta^{\prime}}\right)$.

By pigeonholing on the size of $\left\|\left|e^{i t \phi_{\epsilon_{1}}} f_{\epsilon_{1}} e^{i t \phi_{\epsilon_{1}}} g_{\epsilon_{1}}\right|^{1 / 2}\right\|_{L^{6}\left(Q_{j}\right)}$, we sort the fraction $\sim$ $1 /(\log R)$ of cubes $Q_{j}$ such that

$$
\left\|\left|e^{i t \phi_{\epsilon_{1}}} f_{\epsilon_{1}} e^{i t \phi_{\epsilon_{1}}} g_{\epsilon_{1}}\right|^{1 / 2}\right\|_{L^{6}\left(Q_{j}\right)} \text { is essentially constant. }
$$

We denote by $\mathcal{Q}$ the set of such cubes $Q_{j}$. Thus, to prove 3.39 , it suffices to show that

$$
\lambda^{3}\left|S_{\lambda}\right| \lesssim R^{c_{1} \delta^{\prime}}\left\|\left|e^{i t \phi_{\epsilon_{1}}} f_{\epsilon_{1}} e^{i t \phi_{\epsilon_{1}}} g_{\epsilon_{1}}\right|^{1 / 2}\right\|_{L^{6}\left(\bigcup_{Q_{j} \in \mathcal{Q}}^{6} Q_{j}\right)}^{6}
$$


for some constant $c_{1}$. Indeed, by $(3.37)$, we have $\left|X_{\lambda}\right|^{1 / 3} \lesssim r^{1 / 3}\left|S_{\lambda}\right|^{1 / 6}\left|S_{\lambda}\right|^{1 / 6}$ and $r^{1 / 3}\left|S_{\lambda}\right|^{1 / 6}$ $\leq(\log R)^{1 / 6}(r M R)^{1 / 6}$ by 3.38$)$. Hence, we have

$$
\lambda^{1 / 2}\left|X_{\lambda}\right|^{1 / 3} \leq(\log R)^{1 / 6}(r M R)^{1 / 6}\left(\lambda^{3}\left|S_{\lambda}\right|\right)^{1 / 6} .
$$

Combining this with (3.41), we obtain

$$
\lambda^{1 / 2}\left|X_{\lambda}\right|^{1 / 3} \lesssim r^{1 / 6} M^{1 / 6} R^{1 / 6+c \delta^{\prime}}\left\|\left|e^{i t \phi_{\epsilon_{1}}} f_{\epsilon_{1}} e^{i t \phi_{\epsilon_{1}}} g_{\epsilon_{1}}\right|^{1 / 2}\right\|_{L^{6}\left(\bigcup_{Q_{j} \in \mathcal{Q}} Q_{j}\right)} .
$$

By applying Proposition 3.9 to the right-hand side, we get the desired bound 3.39 and hence 2.11.

It remains to show (3.41). Let $Q^{\prime} \subset Q_{j}$ and let $\psi_{Q^{\prime}} \in \mathcal{C}_{0}^{\infty}\left(\mathbb{R}^{3}\right)$ such that $\mathcal{F}\left(\psi_{Q^{\prime}}\right)$ is supported on $B^{3}(0,2 r)$ and $\psi_{Q^{\prime}}$ decreases rapidly outside $Q^{\prime}$. Since $\widehat{f}, \widehat{g}$ are supported on $B^{2}\left(\xi_{0}, r\right)$, we see that

$$
\mathcal{F}\left(U_{\alpha} f U_{\alpha} g\right)\left(\xi, \xi_{3}\right)=\mathcal{F}\left(U_{\alpha} f U_{\alpha} g\right)\left(\xi, \xi_{3}\right) \mathcal{F}\left(\psi_{Q^{\prime}}\right)\left(\xi, \xi_{3}\right)
$$

Since $\psi_{Q^{\prime}}$ decreases rapidly outside a ball of radius $r^{-1}, \int U_{\alpha} f U_{\alpha} g((x, t)-(y, s)) \psi_{Q^{\prime}}(y, s)$ $d y d s$ is negligible when $|(y, s)| \geq R^{a} r^{-1}$ for sufficiently small $a$. Hence, we have

$$
\sup _{(x, t) \in Q^{\prime}}\left|U_{\alpha} f(x, t) U_{\alpha} g(x, t)\right| \lesssim r^{3} \int_{R^{a} Q^{\prime}}\left|U_{\alpha} f(x, t) U_{\alpha} g(x, t)\right| d x d t+E_{N}
$$

where $E_{N}=O\left(R^{-N}\|f\|_{2}\|g\|_{2}\right)$ for sufficiently large $N$. By applying Lemma 3.3 with $\rho=\epsilon_{1}$ and $\xi_{0}=\eta_{0}$, we get

$$
\left|Q^{\prime}\right| \sup _{(x, t) \in Q^{\prime}}\left|U_{\alpha} f(x, t) U_{\alpha} g(x, t)\right| \lesssim \int_{\widetilde{A}_{Q^{\prime}}}\left|e^{i t \phi_{\epsilon_{1}}} f_{\epsilon_{1}}(x) e^{i t \phi_{\epsilon_{1}}} g_{\epsilon_{1}}(x)\right| d x d t+E_{N}
$$

where $\widetilde{A}_{Q^{\prime}}=\mathcal{A}_{\eta_{0}}^{\epsilon_{1}}\left(R^{a} Q^{\prime}\right)$. By applying Hölder's inequality, we have

$$
\left|Q^{\prime}\right|^{1 / 3} \sup _{(x, t) \in Q^{\prime}}\left|U_{\alpha} f(x, t) U_{\alpha} g(x, t)\right| \lesssim R^{c a}\left(\int_{\widetilde{A}_{Q^{\prime}}}\left|e^{i t \phi_{\epsilon_{1}}} f_{\epsilon_{1}}(x) e^{i t \phi_{\epsilon_{1}}} g_{\epsilon_{1}}(x)\right|^{3} d x d t\right)^{1 / 3}+E_{N}
$$

for some constant $c>1$. If $a$ is sufficiently small, then $\widetilde{A}_{Q^{\prime}}$ is contained in a cube of side length $R^{1 / 2}$; hence, we may assume that $\widetilde{A}_{Q^{\prime}} \subset Q_{j}$. Taking the third power and summing over all $Q^{\prime}$ such that $\widetilde{A}_{Q^{\prime}} \subset \bigcup_{j} Q_{j}$ and using (3.40), we have (3.41) with a minor error term $E_{N}$. This completes the proof.

Remark 3.11. Without difficulty, we can see that the result of Theorem 1.1 extends to the solution of the general dispersive equation. Let $\alpha>1$ and $\Phi$ be a smooth function such that $|\nabla \Phi(\xi)| \geq C^{-1}|\xi|^{\alpha-1}$ and $\left|\partial_{\xi}^{\gamma} \Phi(\xi)\right| \leq C|\xi|^{\alpha-|\gamma|}$ for any $\gamma$ for some constant $C>0$. The reduction to the bilinear estimate in Theorem 2.6 (see Section 2) via polynomial partitioning is nearly identical. Since $\Phi$ is positive definite, by rescaling and Lemma 3.2 . we only need to consider $e^{i t \phi}$ for $\phi \in \mathcal{P}\left(\epsilon_{0}\right)$ (see $(3.42)$ ). Then, by following the proof of Theorem 2.6, we can obtain 2.11) for $e^{i t \Phi}$ in place of $U_{\alpha} f$. 
Remark 3.12. A modification of the proof of Theorem 1.1 combined with the argument in [12] provides 1.2 for $d \geq 3$ whenever $s>d /(2 d+2)$.

\section{References}

[1] J. Bourgain, Some new estimates on oscillatory integrals, in: Essays on Fourier Analysis in Honor of Elias M. Stein (Princeton, NJ, 1991), 83-112, Princeton Math. Ser. 42, Princeton Univ. Press, Princeton, NJ, 1995.

[2] _ On the Schrödinger maximal function in higher dimension, Proc. Steklov Inst. Math. 280 (2013), no. 1, 46-60.

[3] _ A note on the Schrödinger maximal function, J. Anal. Math. 130 (2016), 393-396.

[4] J. Bourgain and C. Demeter, The proof of the $l^{2}$ decoupling conjecture, Ann. of Math. (2) 182 (2015), no. 1, 351-389.

[5] A. Carbery, Radial Fourier multipliers and associated maximal functions, in: Recent Progress in Fourier Analysis (El Escorial, 1983), 49-56, North-Holland Math. Stud. 111, North-Holland, Amsterdam, 1985.

[6] L. Carleson, Some analytic problems related to statistical mechanics, in: Euclidean Harmonic Analysis (Proc. Sem., Univ. Maryland, College Park, Md., 1979), 5-45, Lecture Notes in Math. 779, Springer, Berlin, 1980.

[7] C. H. Cho, S. Lee and A. Vargas, Problems on pointwise convergence of solutions to the Schrödinger equation, J. Fourier Anal. Appl. 18 (2012), no. 5, 972-994.

[8] M. G. Cowling, Pointwise behavior of solutions to Schrödinger equations, in: Harmonic Analysis (Cortona, 1982), 83-90, Lecture Notes in Math. 992, Springer, Berlin, 1983.

[9] B. E. J. Dahlberg and C. E. Kenig, A note on the almost everywhere behavior of solutions to the Schrödinger equation, in: Harmonic Analysis (Minneapolis, Minn., 1981), 205-209, Lecture Notes in Math. 908, Springer, Berlin, 1982.

[10] X. Du, L. Guth and X. Li, A sharp Schrödinger maximal estimate in $\mathbb{R}^{2}$, Ann. of Math. (2) 186 (2017), no. 2, 607-640.

[11] X. Du, L. Guth, X. Li and R. Zhang, Pointwise convergence of Schrödinger solutions and multilinear refined Strichartz estimates, Forum Math. Sigma 6 (2018), Paper No. e14, 18 pp. 
[12] X. Du and R. Zhang, Sharp $L^{2}$ estimates of the Schrödinger maximal function in higher dimensions, Ann. of Math. (2) 189 (2019), no. 3, 837-861.

[13] L. Grafakos, Modern Fourier Analysis, Third edition, Graduate Texts in Mathematics 250, Springer, New York, 2014.

[14] L. Guth, A restriction estimate using polynomial partitioning, J. Amer. Math. Soc. 29 (2016), no. 2, 371-413.

[15] _ Restriction estimates using polynomial partitioning II, Acta Math. 221 (2018), no. 1, 81-142.

[16] S. Lee, On pointwise convergence of the solutions to Schrödinger equations in $\mathbb{R}^{2}$, Int. Math. Res. Not. 2006 (2006), Art. ID 32597, 21 pp.

[17] R. Lucà and K. M. Rogers, Coherence on fractals versus pointwise convergence for the Schrödinger equation, Comm. Math. Phys. 351 (2017), no. 1, 341-359.

[18] _ A note on pointwise convergence for the Schrödinger equation, Math. Proc. Cambridge Philos. Soc. 166 (2019), no. 2, 209-218.

[19] C. Miao, J. Yang and J. Zheng, An improved maximal inequality for $2 D$ fractional order Schrödinger operators, Studia Math. 230 (2015), no. 2, 121-165.

[20] A. Moyua, A. Vargas and L. Vega, Restriction theorems and maximal operators related to oscillatory integrals in $\mathbb{R}^{3}$, Duke Math. J. 96 (1999), no. 3, 547-574.

[21] P. Sjölin, Regularity of solutions to the Schrödinger equation, Duke Math. J. 55 (1987), no. 3, 699-715.

[22] T. Tao, A sharp bilinear restrictions estimate for paraboloids, Geom. Funct. Anal. 13 (2003), no. 6, 1359-1384.

[23] T. Tao and A. Vargas, A bilinear approach to cone multipliers II: Applications, Geom. Funct. Anal. 10 (2000), no. 1, 216-258.

[24] L. Vega, Schrödinger equations: Pointwise convergence to the initial data, Proc. Amer. Math. Soc. 102 (1988), no. 4, 874-878.

Chu-Hee Cho and Hyerim Ko

Department of Mathematical Sciences and RIM, Seoul National University, Seoul 151-747, South Korea

E-mail addresses: akilus@snu.ac.kr, kohr@snu.ac.kr 